QK229

F27P

\title{
LIBRARY
}

ANNEX

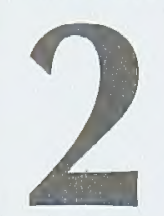




\section{DATE DUE}

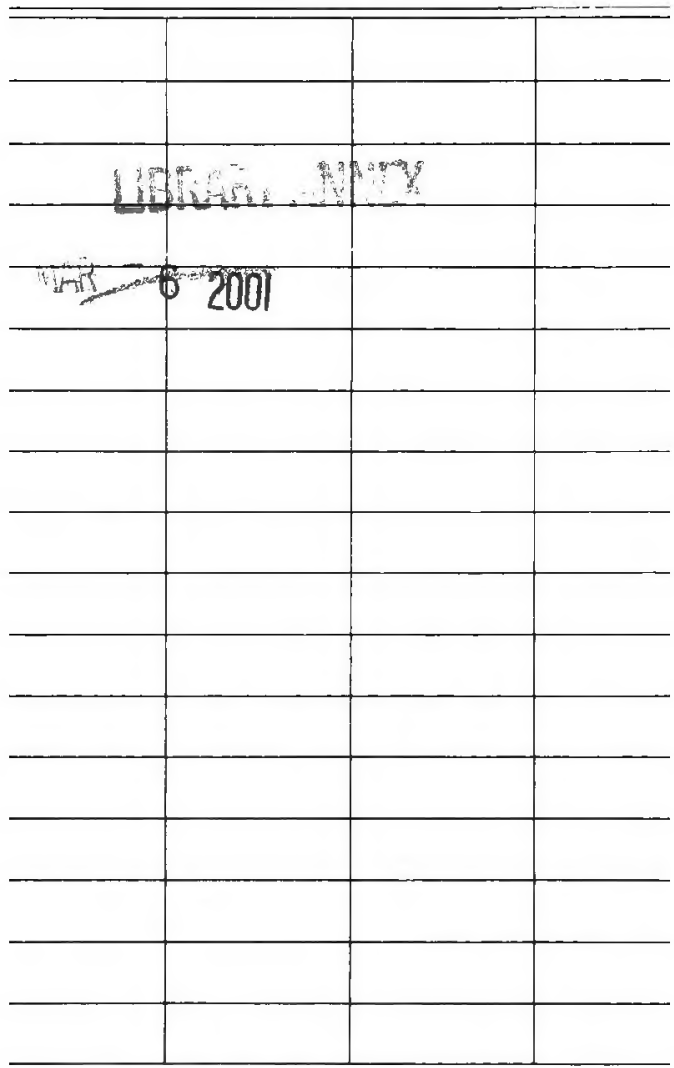

DEMCO 38-297

\section{Ifibrary}

Cornell University Library

QK 229.F27p

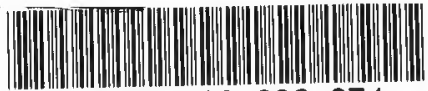

31924000603674 


\section{A PROVISIONAL LIST}

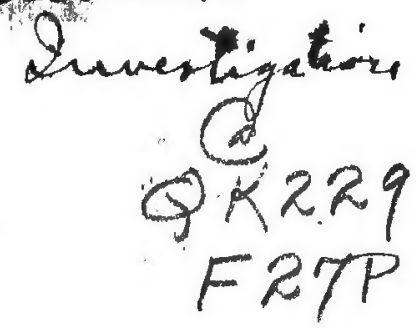

OF THE

INDIGENOUS AND NATURALISED

\section{FLOWERING PLANTS}

OF

\section{JAMAICA}

BY

WILLIAM FAWCETT, B. Sc., F. L.S.

Director of Public Gardens and Plantations,

Jamaica.

KMNGSTON :

ASTON W GARDNER \& CO.

Printers, Publishers, Stationers \& Bookbinders.

127, Harbour Street. 


\section{Cornell University Library}

The original of this book is in the Cornell University Library.

There are no known copyright restrictions in the United States on the use of the text.

http://www.archive.org/details/cu31924000603674 


\title{
A PROVISIONAL LIST
}

\author{
OF THE
}

INDIGENOUS AND NATURALISED

\section{FLOWERING PLANTS \\ $\mathrm{OF}$ \\ J A M A ICA}

BY

WILLIAM FAWCETT, B. Sc., F. L.S.

Director of Public Gardens and Plantations,

Jamaica.

KINGSTON :

$\triangle S T O N$ W GARDNER \& Co.

Printers, Publishers, Stationers \& Bookbinders.

127, Harbour Street. 


$$
\begin{aligned}
& \text { Q } \\
& \text { FR29 } \\
& \text { F27P }
\end{aligned}
$$

250998 


\section{$P R E F A C E$.}

TO those engaged in the study of the Jamaica Flora, it is useful to have 1 a simple list of the plants arranged in systematic order.

The basis of this list is Grisebach"s "Flora of the British West Indian Islands "published in 1864. Since that time the monumental work of Bentham and Hooker, the "Genera Plantarum," (A.D. 1862-1883) has been completed, which renders necessary a revision of the names used by Grisebach. Reference has also been made to many other works, amongst which may be mentioned the later volumes of DeCandolle's "Prodromus" and "Monographiæ Phanerogamarum." But with a very small botanical library at hand, it has not been possible to consult all the works necessary. The list might have been increased by additional names, if opportunity had been afforded for consulting the Herbaria of the British Museum and the Royal Gardens, Kew.

To facilitate reference to Grisebach's Flora, still the standard work, the page on which the plant is described is placed after the name, and where the name has been altered, Grisebach's name is indicated in brackets.

The plants that are not considered indigenous, but have in one way or another been introduced, and have become naturalised, are marked by an asterisk before the name.

On page 50, some names that were omitted, have been added, and it will be found advisable to insert these in their proper places as indicated by the numbers before the names.

\section{Cinchona.}

August, 1893. 



\section{A PROVISIONAL LIST}

OF THE

\section{INDIGENOUS AND NATURALISED FLOWERING PLANTS oF JAMAICA.}

\section{Class I.-DICOTYLEDONES.}

\section{DIVISION I.-POLYPETALAE.}

\section{SERIES I.-THALAMIFLORAE.}

Ranunculace 2 .

Clematis

1. C. dioica, Lim. 1

Ranuncrlus

2. R. repens, $\operatorname{Lim} .1$

3. R. parviflorus, Linn. 2

\section{Dillentace 必.}

Tetracera

4. T. volubilis, Limn. 2

Davilla

5. D. rugosa, Poir. 2

\section{ANONACEE.}

\section{Anona}

6. A. murieata, Linn. 4

7. A. montana, Macf. 4

8. A. laurifolia, Dun. 4

9. A. palustris, Linn. 5

10. A. sericea, Dun. 5

11. A. squamosa, Linn. 5

12. A. Cherimolia, Mill. 5

13. A. reticulata, Linn. 5
Xylopia

14. X. jumaicensis, Griseb. 6

15. X. glalura, Limu. 6

16. X. grandiflora, $A \mu b l, 6$

Boreagea

17. B. virgata, $B$. ‘ $H$. (Oxandra) 7

18. B. laurifolia, B.\& $H$. (Oxandra) 7

\section{Cananga}

19.* (. odorata, Hook. f. \& T. 7

\section{Menispermace.t.}

Cocculus

20. C. domingensis, DC. 10

Cissampelos

21. C. Pareira, Limn. 10

\section{NYMPH代ACE正.}

Nymphæa

2.2. N. ampla, $D C .11$

23. N. blanda, C.F. W. Mey. 11 
Nelumbium

24. N. luteum, Irilld. 12

Papaveracee.

\section{Argemone}

25. A. mexicana, Lim. 13

\section{Boceonia}

26. B. frustescens, Linn. 13

\section{Crucifere.}

Cardamine

27. C. hirsuta, Lim 13

Nasturtium

28. N. officinale, $R \cdot B r \cdot 13$

Sisymbrium

29.*S. officinale, Scop. 13

Capsella

30.* C. Bursa-pastoris, Moench. 14

Lepidium

31. L. virginicum, Limn. 14

32.*L. sativum, Limn. 14

\section{Senebiera}

33. S. didyma, Pers. 14

\section{Cakile}

34. C. æqualis, L'Hér. 14

\section{('APPARIDEA.}

\section{Cleome}

35. C. prentaplaylla, Limn. 15

36. C. speriosa, $H . B$ K. 1 io

37. U. pungeres, Willel. 15

38. C. Houstoni, R. Br. 16

39. C. polygama, Lim. 16

40. C. proctumbens, Jucq. 16

41.*C. visensa, Lim 16

\section{Toraria}

42. T. pendula, Rui: \& Pav, 17

Cratreva

43. C. gynandra, Linn. 17

44. C. Tapia, Linn. 17

Capparis

45. C. ferruginea, $\operatorname{Linn} 17$

46. C. amygdalina, Lam 17

47. C. jamaicensis, Jac\% 18

48. C. cynophallophora, Linn, 18

49. C. frondosa, Jacq. 19

\section{Violariem.}

Viola

31.*T. tricolor, Lim. 26

51." V. Patrini, $D C$.

\section{CANELLACE.}

Caucliat

5:. C. alba, Murr. 109

Cinmamodendron

5.3. C. conticosum, Miers. 109

\section{Bisinee.}

Bixa

5t. B. Orellana, Lim. 20

Lætia

55. L. Thamnia, $S_{m}, 20$

Xylosma.

56. X. nitidum, 4. Gr. 21

57. X. schaefferioides, $A$. Gr .

$$
\text { Pulvide.e. }
$$

Polygala

58. P. paniculata, Lim. 28

59. P. angustifolia, H.B.K. 28 


\section{List of Jamaica Flowering Plants.}

Badiera

60. B. diversifolia, DC. 29

Securidaca

61. S. virgata, $S w .30$

62. S. Lamarckii, Griseb. 30

63. S. Brownii, Griseb. 30

\section{Caryophylle 灰.}

Silene

64.*. S. gallica, Linn. 55

65.*S. Armeria, Linn. 55

Cerastium

66.*C. viscosum, Linn. 55

Stellaria

67.*S. media, Vill. 55

Arenaria

68. A. diffusa, Elll. 55

Drymaria

69. D. cordata, H'ill. 56

Portulace as.

Portulaca

70. P. oleracea, Linn. 57

71. P. pilosa, Linn. 57

7:. P. halimoides, Lim. 57

Talinum

73. T. triangulare, Willd. 56

\section{HYPERICINE 庄.}

Asejrum

74. A. hypericoides, Linn. 112

Clusia

75. C. rosea, Linn. 107
76. C. flava, Linn. 107

Tovomita

77. T. havetioides, Griseb. 106

Symphonia

78. S. globulifera, Limn. $f$.

(Moronobea coccinea) 107

Calophyllum

79. C. Calaba, Jacq. 108

Mammea

80. M. americana, Limn. 108

Rhueedia

81. R. Iaterifiora, Lim.

(Mammea humilis) 108

Quiin:

82. Q. jamaicensis, Griseb. 105

TERNSTRTIIAOEXI.

Maregratria

83. M. umbellata, Lim. 110

Ternstrœemia

84. T. elliptica, Sw. 103

85. T. obovalis, Rich. 103

Cleyera

86. C. theoides Planch. 103

Freziera

87. F. hirsuta, Sim. 104

Laplacea

88. L. hæmatoxylon, Camb. 104

89. L. villosa, Griseb. 104

\section{MaLVACEe.}

Malvastrum

90. M. spicatum, A. Gr. 72.

91. M. tricuspidatum, A. Gr. 72 . 
Anoda

9.2. "A. hastata, Cav. 73.

Sida

93. S. carpinifolia, Lim. 73

94. S. glomerata, Cav. 73

95. S. ciliaris, Linn. 73

96. S. jamaicensis, Lim. 74

97. S. spinosa, Linn. $7 t$

98. S. rhombifolia, Limn. 74

99. S. urens, Linn. 75

100. S. ulmifolia, Cav. 75

101. S. nervosa, DC.7.5

102. S. pyramidata, Cav. 75

103. S. panieulata, Linn. 76

10t. S. cordifolia, Linn. 76

Bastardia

10.. B. viseosa, H.B.K. 80

106. B. bivalvis, H.B.K. 80

Wissadula

107. W. diverwens, B. \& H. T

108. W. rustrata, Mrench.

(A. periplocifolium) 77

\section{Abutilon}

109. A. umbellatum, sint. 78

110. A. indicum, G. Don. 78

111. A. peduneulare, H.B.K. 78

112. A. leionpermum, ririseb. 79

113. A. elatum, Griseb. 79

114. A. striatum, Dicks. 79

Modiola

115. M. caroliniana, $G$, Don. 72

Malachin:

116. M. Mipitata, Limin. 80

117. M. urents, Poit. s1

118. M. ridiatib, Lim. 81

Urena

119. IT. lobata, Limn. 81

120. U. sintiata, Lime 81

Pavonia

121. P. typhaleat, r'w. S:
122. P. spinifex, Cros. 82

123. P. racemosa, $S w$. 83

124. P. corymbosa, Willd. 83

Malvaviscus

12.5. M. arboreus, Cav. 83

Kosteletzkya

136. K. pentasperma, Griseb. 83

Hibisens

127."H. Abelmosehus, Linn.

(Abelmoschus moschatus) 48

128.*H. esculentrus, Linn.

(Abelmoschus) 84

129. H. bifurcatus, C'(w) 84

130. H. clypeatus, Lim!. 85

131. H. trilobus, C're. 85

132." H. vitifolius, Limm. S5)

133. H. phoniceus, Willd. 85

13t. H. Baneroftianus, Mreft. 85

135. H. tiliacens, Linn.

(Paritium) 86

136. H. elatus, Linn.

(Paritium) 86

Therpesia

137.*T. poulnea, c'm. 87

Gros:ipium

138. G. barbidenese, Linue 86

Eriodendron

1:3. E. anfractuosum, $D C^{\prime} .88$

(Nhroma

140. O. Litgenus, sir. 88

\section{Sterc'ItIIAK'E.}

Struevulia

1+1.*S. "anthanguensis, Cun. 89

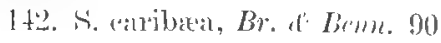

Culi

143. *C. acuminata, Irr. \& Lerm. 90 


\section{List of Jamaica Flowering Plants.}

\section{Helicteres}

144. H. jamaicensis, Jacq. 89

Melochia

145. M. pyramidata, Linn. 93

146. M. tomentosa, Linn. 93

147. M. serrata, Benth. 93

148. M. nodiflora, s'r. 94

149. M. lupulina, Sic. 94

150. M. venosa, sie. ?t

\section{Waltheria}

151. W. americuna, Linu. 9.

\section{Guazuma}

152. G. tomentosa, H.B.K. 90

153. G. ulmifolia, Lam. 91

\section{Theobroma}

154.*T. Cacao, Lim. 91

\section{Ayenia}

155. A. magna, Limn. 91

156. A. lævigata, Suc. 91
157. A. pusilla, Linn. 92

\section{TILIACE}

Triumfertir

155. T. Lappula, Linn. 95

159. T. semitriloba, Linn. 96

160.*T. rhomboidea, .Jacq. 96

161. T. hispida, A. Rich. 96

\section{Corehorus}

162.*C. olitorius, $\operatorname{Linn} .97$

103. C. æstuans, Limn. 97

164. C. acutangulus, Lam. 97

165. C. siliquosus, Linn. 97

166. C. hirsutus, Linn. 97

Muntingia

167. M. Calabura, Linn. 98

Sloanea

168. S. jamaicensis, Hook. 98

\section{SERIES II.-DISCIFLORAE.}

LINEE.

Erythroxylon

169. E. brevipes, DC. 113

170. E. obovatum, Macf. 113

171. E. areolatum, Linn. 113

172. E. squamatum, Vahl. 114

\section{MALPIGHIACEA.}

Byrsonima

173. B. cinerea, DC. 114

174. B. coriacea, DC. 114

\section{Malpighia}

175. M. glabra, Linn. 116

176. M. punicifolia, Linn. 116

177. M. fucata, Ker. 117

178. M. oxycocea, Griseb. 117
Bunchosia

179. B. Lindeniana, Ad. Juss. 115

180. B. media, $D C .115$

181. B. Swartziana, Griseb. 115

Heturopterys

182. H. parviflora, DC. 119

183. H. laurifolia, Ad. Juss. 119

184. H. macrostachya, $A d$. Juss. 120

Brachypterys

185. B. borealis, Ad. Juss. 117

Stigmaphyllon

186. S. ciliatum, Ad. Juss. 118

187. S. emarginatum, $\boldsymbol{A} d$. Juss. 118

188. S. diversifolium, $A d$. Juss. 119

189. S. periplocifolium,

Ad. Juss. 119

\section{Triopterys}

190. T. ovata, Cav. 120 
Tetrapterys

191. T. inæqualis, Cav. 121 Hiraa

192. H. Simsiana, Ad. Juss. (Maseagnia) 1:1

ZYGOPHTLLEÆ.

Tribulus

193. T. cistoides, Linn. 134

194. T. maximus, Linn. 134

Guiacum

195. G. officinale, Linn. $13 \pm$

Gerantace.e.

Geranium

196.* G. pyrenaicum, Lim. 19:197. G. carolinianum, Linn.

OxaLIS.

198. O. Martiana, Zuct. 133

199. O. corniculata, Linn. 133

\section{RUTACE}

Zanthoxylum

200. Z. elava-Herculis, Limm. 138 201. Z. aromaticum, Willd. 138

202. Z. spinosum, Sw. (Tobinia) 137

203. Z. acuminatum, Sw. (Tolinia) 137

204. Z. emarginatum, $S w$.

(Tobinia) 137

205. Z. coriaceum, B. \& II. (Tobinia) 137

206. 7. spinifex, Jacq. (Fagara microphylla) $1: 37$
(2)7. Z. Pterota, H.B.K.

(Fagara lentiscifolia) 137

Esenbeckia

208. E. pentaphylla, Griseb. 135

Peltostigma

209. P. pteleoides, Walp. 136

\section{Citrus}

210. $\ulcorner$. Aurantium, Lim. 132

211." ¿. medica, Linn. 132

212. ${ }^{*}$. decumana, Linn. 132

\section{Annyris}

213. A. Plumieri, DC. 174 214. A sylvatica, Jacq. 174

215. A. balsamifera, Linn. 174

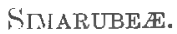

Simaruba

216. S. officinalis, Macf. 139

Picræna

217. P. excelsa, Linrl. 140

Brunellia

218. B. comocladifolia, Humb. \& Bon? 139

Spathelia

219. S. simplex, Lim, 140

Pieramnia

220. P. pentaudra, Sw. 140

221. P. Antidesma, Sw. 141

Picrodendron

222. P. Juglans, ririsel. 177

\section{OCHNACEA.}

Gomplia

223. G. laurifolia, s'tr. 104

224. G. guianensis, Rich. 105

225. G. nitida, S'心. 105 


\section{List of Jamaica Flowering Plants.}

Burseracere.

\section{Bursera}

226. B. simplicifolia, $D C$.

227. B. gummifera, . Turq. 173

Protium

228. P. guianem .e, March.

(Icica heptaphylla) 173

\section{Meliadee.}

Melia

229.*M. Azederach Linn.

(M. sempervirens) 128

Guarea

230. G. trichilioides, Linn.

(G. Swartzii, DC.) 131

Trichilia

231. T. terminalis. Jacq. (Acrilia) 1:?

232. T. hirta, Linn. 129

233. T. spondioides, Ser. 129

23t. T. odorata, $A$ udr.

(Portesia ovata) 130

235. T. Jamaicensis, C. DC.

236. T. mosehata, Sw.

(Moschoxylum Swartzii) 130

237. T. Distini, A. DC.

Swietenia

238. S. Mahagoni, Linn. 131

Cedrela

239. C. odorata, 7inn. 131

\section{OLACINE更。}

Ximenia

240. X. americana, Limn. 310 Schœpfia

241. S. arborescens, Ram. and Schult. 310
242. S. chrysophylloides, Planch. (S. Marchii) 310

Mappia

243. M. rieemosa, Jacq. 310

\section{ILICLNEA.}

\section{Ilex}

241. I. subtriflora, Griseb.

245. I. montana, Griseb. 147

246. I. nitida, Maxin.

217. I. obcordata, $S w .147$

\section{Celastrinez.}

Myginda

248. M. Uragoga, Jacq. 146

249. M. latifolia, $S w .146$

S'chæfferia

250. S. frutescens, Jacq. 146

Elcoodendron

251. E. attenuatum, Rich. 145

252. *E. glaucum, Pers. 146

Hippocratea

2533. H. ovata, Lam. 148

254. H. malpighifolia, Rudge. 148

\section{RHAMNE正.}

Zizyphus

25.i. Z. Chloroxylon, Oliv.

Condalia

256. C. ferrea, Griseb. 100

Sarcomphalus

257. S. laurinus, Griseb. 100

Rhamnus

258. R. sphærospermus, $S w$.

(Frangula) 99 


\section{Colubrina}

259. C. ferruginosa, Brongn. 100 260. C. reclinata, Brongn. 101 261.* C. asiatica, Brongn. 101

\section{Gouania}

262. G. doningensis, Limn. 100

\section{Adrelide..}

Vitis

203. V. caribæa, DC. 102

\section{Ampelocissus}

264. A. Robinsonii, Planch.

Cissus

265. C. sicyoides, Lim. 102

266. C. acida, Lim. 102

267. C. microearpa, Vahl. 102

\section{SAPINUACEA.}

Serjania

268. S. mexicana, Willt. (S. paniculata) 123

269. S. lucida, Schum. 123

270. S. divaricata, $S w .123$

271. S. augustifolia, Willd. 123

Cardiospermum

272. C. Halicacabum, Linn. 122

273. C. grandiflorum, $S w .122$

\section{Paullinia}

274. P. barbadensis, Jacq. 124

275. P. curassavica, Jacq. I24

Schmidelia

276. S. Cominia, Sw. 126

277. S. glabrata, H.B.K. 126

\section{Cupania}

278. C. glabra, $S w .125$
279.*C. Akeesia, Camb.

(Blighia sapida) 125

Ratonia

280. R. apetala Griseb. 126

Melicocea

281.*M. bijuga, Limn. 126

Sapindus

23:. S. Saponaria, Linu: 126

283. S. inæqualis, $D C \cdot 126$

Hypulate

'84. H. trifoliata, $S t e \cdot 12 \overrightarrow{7}$

2s.5. H. paniculata, ('rmll) 127

Dodonæa

286. D. viscosa, Linn. 127

Alvaradoa

287. A. jamaicensis, Benth. 141

Turpinia

288. T. occidentalis, Don. 128

ANACARDIACE正.

Mangifera

289.*M. indica, Linn. 176

Anacardium

290. A. occidentale, $\operatorname{Lin} .176$

Spondias

291. S. purpurea, Limn. 175

292. S. luten, Lim. 175

Comocladia

293. C. integrifolia, Jacq. 175

294. C. pubescens, $E n g l$.

295. C. dentata, Jacq.v. propinqua,

Engl. (C. propinqua) 175

Metopium

296. M. Linnæi, Engl. (Rhus Metopium) 175 


\section{SERIES III.-CALYCIFLOR FE.}

MORTNGEE.

Moringa

297.*M. pterygosperma, $\mathrm{C}^{\prime} \mathrm{c} / \mathrm{m} / \mathrm{l} .16$

\section{ConNarace任.}

\section{Rourea}

298. R. glabra, H.B.K. 228

\section{LEGUMINOSA.}

\section{Crotalaria}

299. C. pterocaulon, Desv. 178

$300 .{ }^{*}$ C. verrucosa, Limn. 178

301.* C. sericea, Retz. 179

302.*C. retusa, Linn. 179

303.*C. juncea, Linn. 179

304.*C. tetragona, Roxb. 179

305.*C. nana, Burm. 179

306.*C. fulva, Roxb. 179

307. C. pumila, Ort. 179

308. C. lotifolia, Iimn. 180

309. C. incana, Linn. 180

310. C. striata, DC. 180

311. "C. quinquefolia, Lim. 180

\section{Ulex}

312.*U. europæus, Linn. 180

\section{Trifolium}

313.*T. repens, Linn. 181

314. T. minus, Relhan. (T. filiforme) 181

\section{Indigofera}

315. I. subulata, Vahl. 181 316.*I. tinctoria, Linn. 181 317. I. Anil, Limn. 181

\section{Tephrosia}

318. T. toxicaria, Pers. 182 319.*T. gxandiflora, Pers. 182
320. T. leptostachya, DC. 182 321. T. cinerea, Per's. 182

Sesbania

:3:-.'. crrandiflora, Pers.

(Agati grandiflora) 183

3.:3. S. æegyptiac:a, Pers. 184

32t. S. aculeata, Pers. 184

325. S. exasperata, H. B. K. 184

Brya

326. B. ebenus, DC. 189

Æssehynomene

327. Æ. americana, Linn. 185

Stylosanthes

328. S. procumbens, $S w .188$

329 . S. viscosa, $S w .188$

Arachis

330. A. hypogæa, Linn. 189

Zornia

331. Z. diphylla, Pers. 185

Desmodium

332. D. triflorum, $D C .186$

333. D. barbatum, Benth. 186

334. D. adseendens, DC. 186

335. D. incanum, DC. 186

336. ${ }^{*} \mathrm{D}$. gangeticum, DC. 187

337.*D. cajanifolium, DC. 187

338. D. uncinatum, DC. 187

339. D. axillare, $D C .187$

340. D. scorpiurus, Desv. 187

341. D. tortuosum, DC. 188

342. D. spirale, $D C$. 188

343. D. molle, DC. 188

344. D. umbellatum, DC.

(Dendrolobium) 188

\section{Lourea}

345.* L. vespertilionis, Desv. 185 
Alysicarpus

346.*A. vaginalis, DC. 185

Vicia

317.*V. sativa, Iinn. 189

Lathyrus

348.* L. tingitanus, Linn.

Abrus

349. A. precatorius, Limu. 190

Centrosema

350. C. Plumieri, Benth. 192

351. '. pubescens, Benth. 19:

352. C. virginianum, Benth. 193

Clitoria

353. C. Ternatea, Lim. 192

354. C. glycinoides, DC. 192

Teramnus

355. T, volubilis, Sw. 193

356. T. uncinatus, Sw. 193

357. T. labialis, Spreng. 193

Erythrina

358. E. Corallodendron, Linn。 199

359. E. velutina, Willd. 199

\section{Mucuna}

360. M. pruriens, DC. 198

361. M. urens, DC. 198

362. M. altissima, DC. 198

Galactia

363. G. tenuiflora, W. \& A. 194

364. G. Lockharti, Griseb. 194

365. G. pendula, Pers. 194

Dioclea

366. D. reflexa, Hook. fl. 198

Canavalia

367. C. obtusifolia, DC. 197

368. C. ensiformis, DC. 197

369. C. altissima, Mucf. 197

Phaseolus

370. P. lunatus, Linn. 196
371. P. vulgaris, Linn, 196

372. P. truxillensis, H.B.K. 196

373. P. semierectus, Linn. 197

\section{Vigna}

37t. V. luteola, Benth. 195

37.5. V. vexillata, Benth. 195

Pachyrhizus

376. P. angulatus, Rich. 195

377. P. tuberosus, Syreng.

Psophocarpus

378. P. palustris, Desv.

Dolichos

379.*D. Lablal, Linn. 196

\section{Cajanus}

380.*C. indicus, Spreng. 191

Rhyncosia

381. R. minima, DC. 190

382. R. phaseoloides, DC. 190

383. R. reticulata, $\mathrm{D} C \cdot 190$

Flemingia

384.*F. strobilifera, R. Br. 191

385.*F. stricta, Roxb. 191

Dalbergia

386. D. Amerimnum, Benth. 202

Eeastaphyllum

387. E. Brownei, Pers.

(Hecastophyllum Brownei) 202

Pterocarpus

388. P. Draco, Linı. 201

Platymiscium

389. P. platystachyum, Be'sth. 200

Lonchocarpus

390. L. latifolius, H.B.K. 199

391. L. violaceus, H.B.K. 200

392. L. sericeus, H.B.K. 200

Piscidia

393. P. Erythrina, Linn. 200 


\section{List of Jamaica Flowering Plants.}

Andira

394. A. inermis, H.B.K. 202

Sophora

395. S. tomentosa, Linn. 203

Swartzia

396. S. grandiflora, Willd. 211

Peltophorum

397. P. Linnæi, Benth. 206

Cæsalpinia

398. C. Bonducella, Flem. (Guilandina) 204

399. C. Bondue, Roxb. (Guilandina) 204

400. C. bijuga, $S w .205$

401.*'C. sepiaria, Roxb. 205

402. C. pulcherrima, $5 w .205$

403. C. coriaria, Willd. (Lebidibia) 206

Hæmatoxylon

404. H. campechianum, Limn. 204

Parkinsonia

405. P. aculeata, Linn. 204.

Cassia

406.*C. Fistula, Linn. 206

407.*C. marginata, Roxb. 207

408. C. grandis, Linn. 207

409. C. bacillaris, Linn. 207

410. C. viminea, Limn. $20 \bar{T}$

411. C. bicapsularis, Linn. 207

412. C. lævigata, Willd. 207

413. C. emarginata, Linn. 207

414.*C. siamea, Lam.

(C. florida) 208

415. C. spectabilis, DC. 208

416.*C. glauca, Lam. 208

417. C. biflora, Limn. 208

418. C. alata, Linn. 209

419. C. occidentalis, Limn. 209

420. C. Tora, Linn. 209

421. C. sericea, $S w .209$
422.*C. obovata, Collad. 209

423.*C. Absus, Linn. 210

424. C. rotundifolia, Pers. 210

42.. C. pilosa, Linn. 210

426. C. serpens, Limn. 210

4:7. C. lineata, $S w .210$

4노. C. polyadena, DC. 210

429. C. glandulosa, Limn. 211

430. C. virgata $S w .211$

431. C nictitans, Limn. 211

4:3. C. patellaria, DC. 211

Bauhinia

433. B. porrecta, $S w$.

(Casparea) 213

434. B. aurita, Ait. (Casparea) 213

43.5. B. ungula, Jacq. 214

4:36.* B. variegata, Linn. 214

Tamarindus

437. T. indica, Linn. 213

Hymenæa

438. H Courbaril, Linn. 213

Crudia

439. C. spicata, Willd. 216

Prioria

440. P. copaifera, Griseb. 215

Entada

441. E. scandens, Benth. 216

Adenanthera

442.*A. pavonina, Limn. 217

Prosopis

443. P. juliflora, DC. 217

Neptunia

444. N. oleracea, Lour. 217

445. N. plena, Benth. 218

Desmanthus

46. D. virgatus, Willd. 218

447. D. depressus, H.B.K. 218. 
Mimosa

448. M. viva, Linn. 218

449. M. pudica, Linn. 219

450. M. asperata, Linn. 219

451. M. thyrsoidea, Griseb. 218

\section{Leucæna}

45\%. L. glauca, Benth. 220

\section{Acacia}

453.*A.Catechu, Willd. 220

454. A. sarmentosa, Desv. 221

455. A. villosa, Willd. 221

456. A. macracantha, Humb. \& Bonpl. 221

457. A. tortnosa, Willd. 222

458. A. Farnesiana, Willd. 229

459." A. arabica, Willd. 222

460.*A. concinna, $D C$.

\section{Lysiloma}

461. L. Marchiana, Griseb. 223

\section{Calliandra}

462. C. portoricensis, Benth. 224

463. C. hæmatomma, Benth. 224

464. C. comosa, Benth. 225

\section{Albizzia}

465. A. Julibrissin, Durazz. 223

466. A. Lebbek, Benth. 223

Pithecolobium

407. P. parvifolinm, Benth.

$$
\text { (Acrcia) 220) }
$$

468. P. fragrans, Benth

(Acacia Berteriana) 223

469. P. latifolium, Benth.

(Calliandra) 225

470. P. Saman, Benth. 22,

(Calliandra) 225

471. P. unguis-cati, Benth. 226

472. P. mieradenium, Benth. 226

473. P. filicifolium, Benth. 226

Enterolobium

474. E. cyelocarpum, Griseb. 226
Inga

475. I. vera, Willd. 228

476. I. ingoides, Willd. 228

\section{ROSACEA.}

Chrysobalanus

477. C. Teaco, Limn. 229

Hirtella

478. H. triandra, S'w. 230

Prunus

479. P. occidentalis, Sw. 231

480. P. sphærocarpa, Sw. 231

Rubus

481. R. jamaicenis, Sw. 231

482. R. alpinus, Macf. 232

Fragaria

483. F. vesea, Linn. 232

Rosa

484.* R. lævigata, Mrichx. 231

\section{SAXIFRAge.⿱一土儿.}

\section{Weinmannia}

485. W. glabra, Linn. fil. 303

486. W. hirta, Sw. 303

\section{CRASSULACEE.}

Bryophyllum

487. B. calycinum, Seclisb. 303

\section{RHIZOPHOREA.}

Rhizophora

488. R. Mangle, Linn. 274

Cassipourea

489. C. elliptica, Poir. 274 
JOMBRETACEA.

Terminalia

490 T. arbuscula, $S w$. (Chuncoa) 275

491. T. latifolia, $S w .276$

492.*T. Catappa, Linn. 276

493. T. Buceras, $B . \& H$.

(Bucida) 276

494. T. capitata, $B . \& H$.

(Bucida) 277

\section{Conocarpus}

49.5. C. erecta, Limn. 277

\section{Laguncularia}

496. L. racemosa, Greitu. 276

\section{Combretum}

497. C. Jacquini, Griseb. 275

\section{Myrtace}

\section{Psidium}

498. P. Guajava, Linn. $2 \pm 1$ 499. P. montanum, $S w .242$

500. P. cordatum, Sims. 242

Myrtus

501. M. fragrans, $S w$. (Anamomis) 240

Myrcia

502. M. splendens, $D C .234$

\section{Calyptranthes}

503. C. Chytraculia, $S w .232$

504. C. Syzygium, Sw. 232

505. C. pallens, Griseb. 233

506. C. rigida, $5 w, 233$

507. C. Wilsoni, Griseb. 233

\section{Pimenta}

508. P. vulgaris, Willd. 240

509. P. Pimento, Griseb. 241

510. P. acris, Willd. 241
Eugenia

511. E. alpina, Willd. 236

512. E. buxifolia, Willd. 236

513. E. monticola, DC. 236

514. E. axillaris, Poir. 236

515. E. lateriflora, Willd. 236

516. E. obtusata, Willd. 237

517. E. disticha, DC. 237

518. E. pallens, DC. 237

519. E. virgultosa, $D C .237$

520. E. æruginea, $D C .237$

521. E. Gregii, $D C .238$

522. E. procera, Poir. 238

523. E. Marchiana, Griseb. 238

524. E. ligustrina, Willd. 239

525. E. uniflora, Linn 239

526. E floribunda, West. 239

597. E. Hartii, Kiarsk.

528. E. Caryophyllata, Thunb. (Caryophyllus) 235

529. ‘E. Jambolana, Lam.

$$
\text { (Syzygium) } 235
$$

530. ${ }^{*}$ E. malaccensis, Limn. 235

531.*E. Jambos, Linn. 235

Grias

532. G. cauliflora, Linn. 243

\section{Metastomace}

Acisanthera

533. A. quadrata, Juss. 269

534. A. recurva, Griseb. 269

Nepsera

535. N. aquatica, Naud. 268

Adelobotrys

536. A. adscendens, Triana (Davya adscendens) 265

Meriania

537. M. purpurea, $S w$.

(M. bullifera) 265 


\section{List of Jamaica Flowering Plants.}

538. M. leucantha, Sw.

(M. rosea) 264

\section{Leandra}

539. L. Eggersiana, Cogn.

Conostegia

540. C. procera, D.Don. 253

541. C. Grisebachii, Cogn.

542. C. superba, D.Don. 253

543. C. montana, D. Don. 254

544. C. rufescens, Naud. 253

545. C. subhirsuta, $D C .253$

\section{Charianthus}

546. C. tinifolius, D. Don.

547. C. Fadyeni, Griseb. 264

Tetrazygia

548: T. albicans, Triana

549. T. angustifolia, $D C$. (Miconia) 258

550. T. hispida, Macf.

(Heterotrichum) 251

551. T. pallens, Cogn.

(T. angustiflora, part) 254

Miconia

552. M. macrophylla, Triana

(Diplochita serrulata) 252

iji). M. dodecandra, Cogn.

(D). Swartziana) 252

5it. M. Guianensis, Cogn .

(1). Fothergilla) 2..71

555. M. triplinervis, Ruiz \& Pav.

(M. trinervis) 25

556. M. miultispicata, Naud. 257

5.5. M. impetiolaris, D. Don. 256

558. M. albicans, Triena

(M. holosericea) 256

559. M. eriodonta, $D C$.

560. M. punctata, D. Don.

561. M. lovigata, $D C .257$

50:. M. splendens, Triana

563. M. obovalis, Nruel.

(M. splendens) 20
564. M. prasina, $D C .257$

565. M. trinervia, $D$ Don.

566. M. minutiflora, $D C$.

(M. myriantha) 257

567. M. fulva, DC.

(Eurychænia punctata) 259

568. M. eiliata, $D C$.

(M. racemosa, v. ciliata) 258

569. M. elata, $D C$.

570. M. vulcanica, Naud.

(Cremanium Sieberi) 262

571. M. rubens, Naud.

572. M. tetrandra, Nuud.

(Cremanium) 262

573. M. theæzans, Cogn.

(Cremanium rubens) 261

574. M. rigida, Triana

(Pleurochænia) 260

575. M. quadrangularis, Naud.

(Pleurochænia) 260

Calycogonium

576. C. glabratum, $D C$. 245

577. C. rhamnoideum, Naud. 245

Heterotrichum

578. H. patens, $D$ (?. 251

Mecranium

579. M. amygdalinum, Triana (Cremanium) 261

580. M. purpurascens, Triana

581. M. virgatum, Triana

(Cremanium) 261

Clidemia

582. C. hirta, D. Don. 246

583. C erythropogon, $D C$.

584. C. strigillosa, $D$ (?. 247

585. C. spicata, $D C$. "' 47

586. C. septuplinervia, Cogn.

587. C. Grisebachii, Cogn.

588. C. crossopetala, Cogn.

589. C. plumosa, $D C$.

(C. Berterii) 247 
590. C. umbrosa, Cogn.

(C. latifolia) 247

เ91. C. pilosa, Cogn.

bb2. C. capillaris, Griseb. 249

Henritttea

593. H. ramiflora, $D C .246$

Henriettella

594. H. sessilifolia, Triana

595. H. Macfadyenii, Triana

596. H. fascicularis, Triana (Ossæa) 246

Ossæa

597. O. hirtella, Trianu

598. O. hirsuta, Triana (Clidemia) 248

599. O. mierophylla, Trirna (Clidemia) 248

600. O. glomerata, Triana

601. O. scabrosa, $D C$. (Cliclemia) 18

602. O. Lima, Triana (Clidemia) 249

603. O. asperifolia, Triana

604. O. mierantha, Macf. (Octopleura) 260

Blakea

605. B. trinervia, Limn. 263

Mouriria

606. M. myrtilloides, Poir. 243

\section{LYTHRARIE 平.}

\section{Ammannia}

607. A. latifolia, Linn. 270

\section{Cuphea}

608. C. Parsonsia, R. Br. 269.

609. C. viscosissima, Jacq. 269

610. C. decandra, Ait. 270

611."C. ignea, $A, D C$.
Nesæa

612. N. salicifolia, $H . B . K$. (Heimia) 271

Lawsonia

613.*L. alba, Lam. 271

Punica

61t. ' P. Granatum, Linn. 242

\section{ONAGRARIEAE.}

Jussiæa

615. J. repens, Linn. 242.

616. J. sedoides, H.B.K. 272

617. J. acuminata, Sw. 272

618. J. variabilis, Mey. 272

619. .J. angustifolia, Lam. 273

fi:0. J. suffruticosa, Linn. 273

(j21. J. hirta, Vahl. 273

Lndwigia (Isnardia)

622. L. palustris Ell. 271

623. L. repens, Sw. 271

624. L. microcarpa, Michx. 271

Enothera

625. E. rosea, Ait. 273

626. E. longiflora, Jacq. 273

\section{SAMYDAOEAT.}

Casearia

627. C. sylvestris, $S w .23$

628. C. serrulata, Sw. 23

629. C. stipularis, Vent. 23

630. C. hirsuta, $S w .23$

631. C. hirta, $S w .23$

632. C. ramiflora, Vahl. 24

633. C. nitida, Jacq. 24

634. C. icosandra, $P l$ \& \& $T$. (Thiodia lætioides) '2.)

\section{Lunania}

635. L. racemosa, Hook. 20 
Samyda

636. S. glabrata, $S w .24$

637. S. serrulata, Linn. 25

638. S. villosa. Sw. 25

Homalium

639. H. racemosum, Jacq. 298

\section{LOASE瓜.}

Mentzelia

640. M. aspera, Linn. 298

\section{TURNERAC瓜.}

Turnera

641. T. ulmifolia, Linn. 296

642. T. Pumilea, Linn. 297

643. T. cistoides, Linn. (Piriqueta) 298

\section{PASSIfLORE}

\section{Passiflora}

644. P. minima, Linn. 290

(i45. P. suberosa, Linn. 290

646. P. hirsuta, Linn. 291

647. P. villosa, Macf. 291

648. P. angustifolia, Sw. 291

649. P. lineariloba, Hook. fil. 291

650. P. coriacea, Juss. 292

651. P. regalis, Macf. 292

652. P. rubra, Linn. 292

653. P. sexflora, Juss. 292

6.it. P. triflora Macf. 293

655.*P. laurifolia, Linn. 293

656.*P. edulis, Sims.

657. P. maliformis, Linn. 293

6.58.*P. quadrangularis, Limn. 293

659. P. ciliata, Ait, 292

660. P. fotida, Linn. 294

661. P. penduliflora, Berter. 294
662. P. oblongata, Sw. 295

663. P. tacsonioides, Griseb. 295

664. P. perfoliata, Linn. 295

665. P. Murueuja, Linn. 295

Carica

666. C. Papaya, Iinn. 290

667. C. prosoposa, Linn. 290

\section{Cucurbitaceæ.}

Lagenaria

668.*L. vulgaris, Ser. 288

Momordica

669. M. Charantia, Linn. 287

670. M. Balsamina, Linn. 287

Luffa

671. L. acutangula, Roxb. 288

Cucumais

672. C. Anguria, Limn. 288

Citrullus

673.*C. vulgaris, Schrad.

Cionosicys

674. ${ }^{* \mathrm{C}}$. pomiformis, Griseb. 288

Sikana

675. S. spherica, Hook, fll., Bot. Mag. 7109

Melothria

676. M. Guadalupensis, Cogn. (M. pervaga) 289

677. M. Fluminensis, Gurdn.

Cayaponia

678. C. racemosa, Cogn. (Cionandra) 286

Sechium

679. S. edule, Sw. 286

Ferillea

680. F. cordifolia, Linn. 289 
BEgONIACEAS.

Begonia

681. B. nitida, Ait. 304

682. B. acuminata, Dryond. 304

643. B. Jamaicensis, $A$. $D C$.

684. B. Purdieanus, A. DC.

685. B. scundens, $S w .305$

\section{Caotem,}

\section{Melocactus}

686. M. communis, DC. 300

\section{Mamillaria}

687. M. simplex, Haw. 300

\section{Cereus}

688. C. Swartzii, Griseb. 3 J1

689. C. eriophorus, Lk. Ott. 301

690. C. repandus, Haw. 301

691. C. triangularis, Haw. 301

692. C. grandiflorus, Haw, :301.

693. C. flagelliformis, Mill. 301

Rhipsalis

694. R. Swartzianus, Pfeiff. 3012 (90). R. Cassytha, Gortn. 302

\section{Opuntia}

696. O. Tuna, Mill. 302

697. O. Ficus-indica, Mill. 30\%

698. O. spinosissima, Mill. :30.)

699. O. coccinellifera, Mill. 302

Pereskia

700. P. amdleata, Mill. 303

Ficoide

\section{Sesuvium}

701. S. portulacastrum, Iinn. 57

Trianthema

702. T. monogynum, Linn. 58

Mollugo

703. M. verticillata, Linn. 56 704. M. nudicaulis, Lam. .56

\section{UMBELLIFERÆ.}

Hydrocotyle

705. H. umbellata, Linn. 307

706. H. asiatica, Linn. 307

Eryngium

707. E. fcetidum, Linn. 308

Apium

708. A: leptophyllum, F. v. Muell. (Helosciadium) 308

\section{Carum}

709.*C. Petroselinum, Benth.

(Petroselinum) 308

Peucedanum

710.*P. sativum, Benth.

(Pastinaca) 308

Ditucus

711. ${ }^{*}$ D. Carota, Linn. 308

ARALIaCEæ.

Sciadophyllum

712. S. Brownei, Spreng. 306

Dendropanax

713. D. pendula, Decne. \& Planch. (Hedera) 306

714. D. wutans, Sw.

(Hedera) 306

71.). D. arboreum, Decne. \& Planch.

(Sciadophyllum Jacquini) 306

Ureopanax

716. O. capitatum,Decne. \& Planch. (Sciadophyllum) 306

\section{CornaceE.}

\section{Garrya}

717. G. Fadyenii, Hook. (Fadyenia Hookeri) 286 


\section{Division II.-GAMOPETAL $\mathbb{E}$.}

\section{SERIES I.-INFERZE.}

CAPRIFOLIACEX.

\section{Viburnum}

718. V. villosum, S2o. 315

719. V. glahratum, H.B.K. 315

\section{RUBIACE}

\section{Macroenemum}

720. M. jamaicense, Lim. 32:?

Manettia

721. M. Lygistum, Sw. 329

722. M. coccinea, Willd. 329

Hillia

7.2:3. H. longiflora, Sw. 325

72t. H. tetrandra, Sw. 325

\section{Exostemma}

725. E. brachycarpum, Roem. \& Schult. 3:-4

726. E. triflorum, G. Don. 324

727. E. caribæum, Roem. \& Schult. 324

\section{('himarrhis}

728. C. cymosa, Jacq. 323

I'ntlandia

7:9. P. grandiflora, Linn. 3:t

730. P. gypsophylla, Macf. 324

731. P. coccinca, Sw. 324

\section{Rondeletia}

732. R. laurifolia, $S w .326$

733. R. racemosa, $S w .326$

7i3t. R. thyrsoidea, Sw. 326

73.5. R. tomentosa, Sw. 321;

736. R. trifoliata, Jacq. 326

737. R. polita, Griseb. 326

793. R. daphnoides, Griseb. 3327

739. R. americina, Linn. 327

740. Ii. hirsuta, Sw. 323
741. R. buxifolia, Vahl. 328

742. R. hirta, $S w .328$

743. R. incana, $\$ w .328$

7H. R. cincta, Griseb. 329

745. R. glauca, Griseb. 329

Rhachicallis

746. R. rupestris, DC. 330

Oldenlandia

747. O. coryubosa, Linn. 330

Lueya

748. L. tuberosa, $D C .331$

Gonzal at

749. G. Petesia, Griseh. 321

Schradera

750. S. cephalotes, Trthl. 319

Sabicea

751. S. hirta, Sw. 32.3

Coccocypselum

75. C. repens, $S w .32$.

753. (C. Pseudotontantea, Griseb. 32.)

Hamelia

754. H. ventricosa, $S u \cdot 320$

755. H. cuprea, $5 w .320$

756. H. chrysantha, $S w .320$

757. H. patens, Jacq. '3:0

758. H. lutea, Rolw. 320

Hoffmannia

759. H. palumenlata, Sw. 321

Catesbra

760. ('. spinosa, Limm. 317

Randia

761. R. aculeata, Lim. : 318

Guettardia

762. (1. longiflora, Griseh. 332 
763. G. argentea, Lam. 332

764. G. scabra, Lam. 332

705. G. elliptica, S'r. 332

766. G. crispiflora, Vahl. 333

Antirxhœa

767. A. bifurcata, $B . \& H$. (Stenostomum) 333

76. A. tomentosa, B. \& H. 333

769. A. nitida, B. \& H. $33: 3$

770. A. lucida, B. \& H. 334

Machaonia

771. M. rotundata, Griseb. 348

77\%. M. cymosa, Griseb. 348

\section{Chiococea}

773. C. racemosa, Jacq. 33';

774. C. parvifolia, Wullschl. $3: 37$

Scolosanthus

775. S. versicolor, Vahl. $\because 35$

\section{Ixora}

776.*I. tenuiflora, Roxb. 338

777.* I. Pavetta, Roxb. 338

\section{Coffea}

778.* C. arabica, Limn. 338

\section{Morinda}

779. M. Royoe, Lim. 347

780.*M. citrifolia, Limn. $3 k 7$

\section{Faramea}

781. F. odoratissima, DC. 338

\section{Psychotria}

752. P. uliginosa, $S w .340$

783. P. grandis, iw. 340

784. P. Mapouria, Roem. \& Schult. $3+0$

785 P. dura, Sm. 340

786. P. marginata, Stc. 340

787. P. glabrata, Siv. 341

788. P. temuifolia, sic. 341

789. P. Brownei, Spreng. 3+1

790. P. androsæmifolia, Griseh. $341_{1}$
791. P. celastroides, Griseb. 341

79... P. dasyophthalma, Griseb. 341

793. P. hirsuta, $S w .341$

7!). P. undata, Jacq. 342

79.). P. rufescens, H.B.K. 342

796. P. lanceolata, Nutt. 342

7!17. P. fcetida, Grisel). 312

798. P. brachiata, Sw. 343

79!. P. pubescens, Sw. 342

800. P. peduneulata, S't. $3 \pm 3$

801. P. corymbosa, $S w .343$

802. P. patens, Sic. 343

803. P. Myrtiphyllum, $S w .3+4$

Palicourea

804. P. crocea, DC. 345

S0.5. P. alpina, UC: 345

806. P. pulchra, Griseb. 345

807. P. Pavetta, DC.:346

Rudgea

ज小S. R. caribæa Benth. 339

Geophila

809. G. reniformis, Don. $3 \pm 7$

Cephaelis

810. C. punicea, Vahl. 346

Ernodia

811. E. litoralis, s's. :; $; 4$

Dimdia

51... U. prostrata. Sw. 348

813. D. sarmentosa, Sw. 349

Spurmacoce

814. S. tenuior, Lam. 349

81.5. ז. lævis, Lam.

(Borrera) 349

816. S. hirta, $S w .3 \pm 9$

817. S. simplex, B.\&H. 350

818. $\therefore$. verticillata, Sw. 350

819. S. spinosa, sic. 3.50

Mitracarpum

8.0). II. villosum, Chum. d schlecht. 350 
Richardsonia

821. R. scabra, St. Hil. 351

Galium

822. G. hypocarpium, Endl. 351

\section{Contpositas.}

Sparganophorus

823. S. Vaillantii, Gartn. 352

\section{Vernonia}

824. V. cinerea, Zess. 35:?

825. V. arborescens, Sw. 353

826. V. acuminata, Less. 353

827. V. rigida, Sw. 354

828. V. tricholepis, $S w .354$

\section{Elephantopus}

829. E. mollis, H. B. K. 355.

830. E. angustifolius, $S w .355$

831. E. spicatus, Juss.

(Distreptus) 350

\section{Rolandra}

832. R. argentea, Rottb. 355

\section{Adenostemma}

833. A. Swartzii, Cass. 356

Ageratum

834. A. conyzoides, Linn. 356

835. A. muticum, Grisel. 356

\section{Eupatorium}

836. E. macrophyllum, Linn.

\section{(Hebeclinium) 356}

837. E. rigidum, $S w .357$

838. E. conyzoides, Vahl. 358

839. E. odoratum, Linn. 358

840. E. heteroclinium, Griseb. 358

841. E. ivifolium, Linn. 359

84\%. E. cordifolium, S2v. 359

\$4\%. E. montanum, sw. 359

8+4. E. tetranthum, Griseb. 360

s.5. E. glandulosum, $I$ H.B.K. 360
846. E. nervosum, Sw. 361

847. E. celtidifolium, Lam. 361

848. E. corylifolium, Griseb. 361

849. E. triste, Griseb. 361

850. E. villosum, $S w .361$

851. E. sehizanthum, Griseb. 361

852. E. paniculatum, Schrad. 362

853. E. Dalea, Limn.

(Critonia) 362

854. E. parviflora, Sw. 362

Mikania

855. M. hastata, Willd. 362

856. M. Swartziana, Griseb. 363

857. M. latifolia, Sm. 363

858. M. gonoclada, $D C .364$

859. M. orinocensis, H.B.K. 364

Brickelia

860. B. diffusa, A. Gr. $350^{\circ}$

Egletes

861. E. domingensis, Cass. 380

Erigeron

862. E. jamaicensis, $S w .364$

80i3. E. rivularis, $S w .365$

S6it. E. strictus, $D C .365$

86.5. E. bonariensi i, Limn. 365

866. E. canadensis, Lum. 365

\section{Baceharis}

si7. B. dioica, Vall. 360

868. B. myrsinites, I'ers. 366

869. B. scoparia, Sw. 366

Pluchea

870. P. odorata, C'tess. 366

871. P. purpurascens, DC. 367

Pterocaulon

872. P. virgatum, DC. 367

Gnaphalium

873. G. albescens, sir. 380

874. G. domingensi, Lam. 380

87.;. G. americanum, Hill. 380 
Clibadium

876, C. asperum, $D C .367$

877. C. terebinthaceum, DC. 368

878. C. Alexandri, Griseb. 368

Acanthospermum

879. A. humile, DC. 369

\section{Parthenium}

880. P. Hysterophorus, Linn. 369

Ambrosia

891. A. artemisifolia, Limn. 370

882. A. psilostachya, DC. 370

Zinnia

883. Z. multiflora, Linn. 370

Enhydra

884. E. sessilis, DC. 369

Eclipta

885. E. alba, Hassk. 370

Isocarpha

886. I. oppositifolia, $R . B r .376$

887. I. angustata, Griseb. 376

Borichia

888. B. arborescens, $D C .371$

Wedelia

889. W. carnosa, Rich. 371

890. W. gracilis, Rich. 371

Eleutheranthera

891. E. ovata, Poit.

(Ogiera) 368

Melanthera

892. M. deltoidea, Rich. 372

Verbesina

893. V. gigantea, Jacq. 374

894. V. alata, Linn. 374

Spilanthes

895. S. uliginosa, Sw. 376

Salmea

896. S. grandiceps, Cass. 375
897. S. sessilifolia, Griseb. 375

898. S. hirsuta, $D C .375$

Chænocephalus

899. C. petrobioides, Griseb. 374

Synedrella

900. S. nodiflora, Gœrtn. 377

Cosmos

901. (. candatus, H.B.K. 373

Bidens

902. B. leucantha, Willd. 373

903. B. pilosa, Limn. 373

904. B. tereticaulis, DC. 373

905. B. bipinnata, Limn. 373

Chrysanthellum

906. C. procumbens, Rich. 377

Graliusoga

907. G. parviflora, Cav. 379

Calea

908. C. jamaicensis, Linn. 379

Porophyllum

909. P. ruderale, Cass. 379

Pectis

910. P. punctata, Jacq. 377

911. P. Swartziana, Less. 378

912. P. linifolia, Less. 378

913. P. ciliaris, Linn. 378

911. P. prostrata, Cav. 378

915. P. Plumieri, Griseb. 378

Chrysanthemum

916.*C. Parthenium, Pers. (Pyrethrum) 380

\section{Liabum}

917. L. Brownei, Cass. 383

N'eurolana

918. N. lobata, R. Br. 381

Erechthites

919. E. hielacifolia, Raf. 381 
Emilia

920.* E sonchifolia, DC. 381

\section{Senecio}

921. S. discolor, $D C .382$

922. S. Swartzii, $D C .382$

923. S. Fadyeni, Griseb. 382

(124. S. laciniatus, DC. 382

925.*S. vulgaris, Limn.

\section{Gynoxys}

926. G. incana, Less. 381

(haptalia (Leria)

927. C. nutans, Hemsl. 383

928. ('. dentata, ('c's.s. 383

929. C. albicans, B. \& H. 383

930. C. pumila, B.\&H. 383

Trixis

931. T. frutescens, $\mathrm{P}, \mathrm{Br} \cdot 383$

\section{Lapsana}

932." L. communis, Lim. 384

Hypochæris

933.*H. glabra, Lim.

\section{Taraxacum}

931.*T. offeinale, Wigg. 384
Lactuca

935. L. jamaicensis, Griseb. 384 Sonchus

936.*S. oleraceus, Linn. 384

937. S. asper. Vill. 385

\section{Goodenoviez.}

Scævola

938. S. Lobelia, Linn.

(S. Plumieri) 388

\section{Campandlac'e无.}

\section{Isotoma}

939. I. longiflora, Presl. 388

Lobelia

940. L. Cliffortiana, Lim. 385

911. L. acuminata, S'r. (Tupa) 386

942. L. ensifolia, $B$. $H$. 398

943. L. caudata, B. d. H. 386

944. L. Martagou, R. \& H. :386

945. L. assurgens, Linn. 387

946. L. conglobata, I.tim. 387

Specularia

947. S. perfoliata, $A, D C$.

\section{SERIES. II.-HETEROIIERAE.}

VACCINLACEÆ.

Vaccinium

948. V. meridionale, sw. 144

\section{ERICACE}

Lyonia

949. L. jamaicensis, Don. 14:

950. L. octandra, Griseb. 142

Clethra

951. C. tinifolia, Sw. 141

952. C. bracteata, Griseb. 141
953. C. Alexandri, Grivel. 14:

\section{Plumbagrnem.}

Plumbago

95t. P. scandens, Limu. 390

\section{Prinulacief.}

Anagallis

95.5.*. A. al've'nsis, Jimn.

Centunculus

956. C. pentandrus, $R . \operatorname{Rr} .390$ 


\section{MrRsLNE}

Mypsine

9.7. M. læta, A. DC. 392

958. M, coriacea, $R . B r .392$

\section{Wallenia}

959. W. laurifolia, Sw. 394

960. W. clusiifolia, Grise?. 394

961. W. venosa, Griseb. 394

\section{Ardisia}

962. A. xylosteoides, Griseb. 395 963. A. clusiodes, Griseb. 396 964.* A. humilis, Vahl. 396 965. A. coriace $S w .396$ 966. A. tinifolia, Sw. 396

\section{Jacquinia}

96‥ J. armillaris, Linn. $: 39 \overline{7}$

968. J. aristata, Jacq. 397

\section{SaPotack...}

\section{Chrysophyllum}

969. C. Cainito, Limn. 398

970. C. oliviforme, $L$ rtir. 398

971. C. microphyllum, Jacq. 398

\section{Lucuma}

972. L. multiflora, A.DC. 402

973. L. mammosa, Gertn. 402

\section{Sideroxylon}

974. S. mastichodendron, Jacq. 399
975. S. floribundum, Griseb. 399

976. s. pomiforme, A. DC.

(Sapota rugosa) 399

Aehras

977. A. Sapota, Linn. 399

Bumelia

9.8. B. retusa, Sw. 401

979. B. cuneata, Sw. 401

980. B. l'stundifolia, Sw. 401

Dipholis

981. D. nigra, Griseb. 400

982. D. salicifolia, A. DC. 401

983. D. montana, Grisel. 401

Mimusons

984. II. globosa, Gertn. 400

9.5.). II. Kauki, Linn. 400

986. M. Silleroxylon, Pierre MIss. (Sapota Sideroxylon) 399

\section{EBENac'E.}

Diospyros

987. D. tetrasperma, $S w .404$

\section{STtrorace.}

Sirmulutin

988. s. octopetala, su.

989. S. Jamaicensis, $\pi r$ \& $\operatorname{tr} b$.

990. s. tubulifera, $K r$ \& \& $L^{r} b$.

\section{SERIES III.-BICARPELLAT E.E.}

\section{Oleace.e.}

\section{Jasminum}

991. J. officinale, Lim. 406 992.* J. simplicifolium, Forst. (J. gracile) 406

\section{Forestiera}

993. F. segregata, $K r$ \& $U v b$. (F. eassinoides \& pornlosa) 406
Osneanthus

991. O. fragrans, Lour. (Olea) 405

Linociera

995. L, incritsita, B. $H$. (Hæuianthus) 405

996. L. ligustrina, $S u \cdot 405$ 997. L. latifolia, Iahl. 


\section{Apocynace .}

Allamanda

998.*A. cathartica, Linn. 407

Rauwolfia

999. R. nitida, Linn. 407

1000. R. canescens, Linn. 407

Thevetia

1001. T. neriifolia, Juss. 407

\section{Cameraria}

1002. C. latifolia, Linn. 410

1003. C. angustifolia, Linn. 410

Vinea

1004. V. rusei, Limn, 410

\section{Plumeria}

1005.*P. rubra, Lim. 411

1006. P. Tenorii, Gasp. 411

1007. P. whtusa, Linn. 411

Tabernæmontana

1008. T. laurifolia, Linn. 409

1009. T. citrifolia, Jacq. 409

1010. T. discolor, Sw. 409

1011. T. Wullschlægelii, Griseb.409

Anechites

1012. A. asperuginis, Griseb. 410

Forsteronia

1013. F. corymbosa, Mey. 412

1014. F. Alexandri, Griseb. 412

1015. F. floribunda, G. Don. 412

1016. F. Wilsonii, $B . \& H$.

(Thyrsanthus) 412

\section{Echites}

1017. E. torosa, Jacq. 413

1018. E. Brownei, Muell. Arg. 414 1019. E. repens, Jacq. 414

1020. E. umbellata, Jacq. 414

Rhabdadenia

1021. R. paluclosin, Murell, Arg. (E) hits) 415

\section{Urechites}

1022. U. suberecta, Muell. Arg.

(E. neriandra \& suberecta) 415

1023. U. jamaicensis, $B . \& H$.

(E. jamaicensis) 416

\section{ASCLEPIADE理.}

C'ryptostegia

1024. "C. grandiflora, R. Br. 416

Philibertia

1025. P. Brownei, B. \& $H$.

(Sarcostemma) 419

Fischeria

1026. F. scandens, $D C$. 421

1027. F. cincti, Griseb. 4 't 1

Oxypetalum

1028. O. riparium, H.B.K. 419

Gomplocarpus

1029.*G. fruticosus, R. $B v .419$

Calotropis

1030.*C. procera, $R . B r .420$

Asclepias

1031. A. curassavica, Limn. 419

10:3. A. nivea, Limu. 419

Metastelma

1033. M. parriflorum, $R . B r .417$

1034. M. albiflorum, Griseb. 417

1035. M. Schlechtendalii,Decre. 417

Vincetoxicum

1036. V. filiforme, $B$. \& $H$. (Amphistelma) +18

1037. V. leptocladum, Decne. (Amphistelma) 418

Roulinia

103i. R. jamaile(nlsis, B. \& II. (Enslinia) 418

(ionolobus

1039. G. strllatus, Grisels. 420 
1040. G. pubescens, Griseb. 420

1041. G. rhannifolius, Griseb. 420

Marsdenia

1042. M. clausa, R. Br. 422

\section{LOGANIACE 现.}

\section{Spigelia}

1043. S. Anthelmia, Linn. 331

\section{Mitreola}

1044. M. petiolata, Torr \& Gr. 331

Polypremum

1045. P. procumbens, Linn. $4: \bar{T}$

Buddleia

1046. B. americana, Linn. $4 \geq 7$

\section{Gentiane.e.}

Voyria

104i. V. uniflora. Lam. 4:J

1048. V. tenella, Guild. 425

1049. V. mexıcana, Griseb. 425

Leianthus

1050. L. longifolius, Griseb. $42 \pm$

1051. L. latifolius, Griseb. 424

1052. L. exsertus, Griseb. 425

1053. L. umbellatus, Griseb. $42 \mathrm{~J}$

\section{Erythræa}

1054.*E. ramosissima, Pers. 422

\section{Schultesia}

1055. S. stenophylla, Mart. 423

105̄6. S. heterophylla. Miq. 423

Eustoma

1057. E. exaltatum, Salisb. 422

Lisianthus

1058. L. thamnoides, Griseb, 42t

Limnanthemum

1059. L. Humboldtianum, Griseb. 426
HYDROPHYLLACEE.

Nama

1060. N. jamaicensis, Linn. 477

Hydrolea

1061. H. spinosa, Linn. 477

\section{BORAGINE \#.}

\section{Cor'dia}

106:\%. (. gerascanthus, Jacq. 478

1063. C. gerascanthoides, H.B.K. 478

106t. C. alba, Rom. \& Schult. 478

1065. C. Sebestena, Jacq. 478

1066. ('. Collococea, Jinn. 478

1067. C. nitida, Vahl. 479

1068. C. elliptica, Sir. 479

1069. C. macrophylla, Mill. 479

1070. C. sulcata, DC. 480

1071. C. ulnifolia, Juss. 480

1072. C. eylindristachya, Rcm. \& Schult. 480

1073. C. martinicensis, Rom. \& Schult. 480

1074. ''. globosa, H. B. K. 481

Bourreria

1075. B. succulenta, Jacq. $4 \$ 1$

1076. B. tomentosa, G. Don. 481

1077. B. domingensis, Griseb. 482

Ehretia

1078. E. tinifolia, Linn, 481

Rochefortia

1079. R. cuneata, $S w .482$

1080. R. acanthophora, Griseb. 48.2

Tournefortia

1081. T. gnaphalodes, $R, B r .483$

1082, T. hirsutissima, Lim. 483

1083. T. filiflora, Griseb. 483

1084. T. "'mosa, Linn. 483

1085. T. astrotricha, $D C .483$ 
1086. T. bicolor. $S w .483$

1087. T. staminea, Griseb. 484

1088. T. laurifolia Vent. 484 1089. T. volubilis, Linn. 484

1090. T. ferruginea, Lam. 484

1091. T. tomentosa, Mill. 485

10!r?. T. poliochros, Spreng. 485

1093. T. incana, Lam. 485

\section{Heliotropium}

1094. H. indieum, Linn. 485

1095. H. parviflorum, Linn. 485

1096. H. inundatum, Sw. 486

1097. H. curassavicum, Lim. 486

1098. H. filiforme, H.B.K. 486

1099. H. fruticosum, Lizn. 486

\section{Convolvulace}

Argyreia

1100.*A. tiliæfolia, Wight. 466

1101.*A. bracteata, Chois. 466

\section{Ipomæa}

1102. I. Bona-nox, Lim. 466

1103. I. tuberosa, Lim $41 \mathrm{li}$

1104. I. sinuata, Orteg. (I. dissecta) 467

110.). I. pentaphylla, Jacq. 467

1106. I. quinquefolia, Griseh. 468 1107."I. Batatas, Lam. 468 1108. I. fastigiata, $S w .468$ 1109. I. sidæfolia, Chois. 468 1110. I. cyanantha, Griseb. 469

1111. I. violacea. Linn. 469

1112. I. setosa, Lindl. 469

1113. I. carnea, Jacq. 469

1114. I. Cavanillesii, Rom. "\& Schult. 470

1115. I. triloba, Linn. 470

1116. I. Carolina, Linn. 470

1117. I. umbellata, Mey. 470

1118. 1. Pes-capræ, Sw. 470
1119. I. asarifolia, Rom. \& Schult. 471

1120. I. acetosæfolia, Rom. \& Schult. 471

1121. I. martinicensis, Mey. 471

1122. I. macrorrhiza, Rom. \& Schult. 471

1123. I. Quamoclit, Linn. 472

1124. 1. coccinea, Linn. 472

1125. I. hederifolia, Linn. 472

1126. I. hederacea, Jacq.

(I. Nil) 473

1127. I. purpurea, Lam. 473

11's. I. acuminata, Roem. \& Schult. 473.

1129. I. jamaicensis, Don 473

1130. I. Thomsoniana, Mast.

1132. I. Horsfallix, Hook.

Jaequemontia

1132. J. violacea, Chois.

(Convolvulus pentanthus) 474

Convolvulus

1133. C. micranthus, Rom. \&: Schult. 474

1134. C. jamaicensis, Jacq. 474

1135. C. nodiflorus, Desr. 475

Evolvulus

1136. E. arbuscula, Poir. 475

11: $:$. E. sericeus, $S w .475$

11:38. E. linifolius, Linn. 475

1139. E mucronatus, $S w .475$

1140. E. nummularius, Limn. 475

Dichondra

1141. D. repens, Forst. 476

(includes D. sericea)

Cuscuta

114.2. C. americana, lim, titi

1143. C. umbellata. H.B.K. 476

11+t. C. obtusiflora, H B.K. 476

11 t.). ('. indecora, Chois. 476 


\section{SollaNaceze.}

\section{Lycopersicum}

1146. L. esculentum, Mill.

(L. Humboldtii) 436

\section{Solanum}

1147. S. nigrum, Linn.

$$
\text { (S. nodiflorum) } 437
$$

1148. S. Seaforthianum, Andr. 437

1149. S. triste, Jacq. 437

1150. S. acropterum, Grisels. $4: 37$

11.51. S. punctulatum, Dun. 437

1153. S. verbascifolium, Linn. 438

1153. S. havanense, Jacq. 438

1154. S. retrofractum, Vahl. 439

11.5. S. racemosum, Linn. 439

1156. S. bahamense, Lim. 441

1157. S. jamaicense, $S u$. $t \pm 1$

1158. S. torvum, $S w .441$

11.59. S. inclusum, Griseb. $4+1$

1160. S. mammosum, Lirn. 4t3

1161. S. aculeatissimum, Jacq. $4^{2}$.

Cyphomandra

116:. C. betacea, Sendtu.

Physalis

1163. P. peruviana, Lim. 435

116it. P. angulata, Linn. 436

(includes var. P. Linkiana)

\section{Bassovia}

116i. B. macrophylla, $B$. \& $H$. (Witheringia) 435

Capsil'um

1166. C. frutescens, $L \mathrm{im} .436$

1167. C. baccatum, Lim. $4: 36$

\section{Acnistus}

1168. A. arborescens, Schlecht. 4i35)

\section{Solandra}

1169. S. grandiflora, $S w .433$

1170. S. longiflora, Sw. 433

\section{Datura}

1171. D. suaveolens, Humb. \& Bonpl. 433

1172. D. Tatula, Limn. 434.

1173.*D. Stramonium, Linn. 434

\section{Cestrum}

1174. C. pallidum, Lam. 43

117.). (. latifolium, Lam. $\$ 3$

1176. C. macrophyllum, Vent. 443

1177. C. hirtum, Sw. 444

1178. ( . nocturnum, Linn. 444

Nicotiana

1179. N. pusilla, Linn. 434

1180. N. Tabacum, Linn. 434

Browallia

1181. B. demissa, Limn. 431

Brunfelsia

11'... B. americana, Sw. 432

1LA3. B. jamaicensis, Griseb. 432

118t. B. nitida, Benth. 432

1185. B. undulata, $S w .432$

\section{S'L'RUPHULARINEA.}

Calevolaria

1186.*C. pinnata, Lim.

Angelonia

1187. A. angustifolia, Benth. 431

Linaria

1188. ${ }^{*}$ L. vulgaris, , Irill. 431

Antirrhinum

1189.*A. Orontium, Linn.

Maurandia

1190.* II. standens, A. Gr.

\section{Stemodia}

1191. '.. maritima, Linn. 429

1192. s. durantifolia, sic. +29 


\section{Ferpestis}

1193. H. chamædryoides,

$$
\text { H.B.K. } 430
$$

1194. H. sessiliflora, Benth. 430

1195. H. repens, Cham. \& Schl. 430 1196. H. Monnieria, H. B. K. 430

\section{Vandellia}

1197. V. diffusa, Limn 430

Scoparia

1198. S. duleis, Liun. 427

\section{Capraria}

1199. C. billora, Linn. 427 (includes C. mexicana)

\section{Veronica}

1200. V. serpyllifolia, Linn. 428

1201. V. peregrina, Linn.

1202. "V. arvensis, Linn.

\section{Buchnera}

1203. B. elongata, $S w .428$

Gerardia

1204. G. hispidula, Mart. 428

\section{LENTIBULARIEA.}

Utricularia

1205. U. pusilla, Vahl. 391

1206. U. obtusa, $S w .391$

Pinguicula

1207. P. elongata, Benjan. 39?

\section{GESNERACEA.}

\section{Achimenes}

1208. A. coccinea, Pers. 459

\section{Gesnera}

1209. G. curymhowa, Sw. 459

Pentarhaphia

I210. P. longiflora, Lindl. 460
1211. P. catalpiflora, Decne. 460

1212. P. calyeosa, Decne. 460

1213. P. calycina, B. \&. $H$.

$$
\text { (Vaupellia) } 460
$$

1214. P. humilis, $B$. \& $H$.

$$
\text { (Conradia) } 461
$$

1215. P. mimuloides, $B$. $H$.

(Conradia) 461

1216. P. neglecta, $B . \& H$.

(Conradia) 461

1217. P. clandestina, $B . \&$. $H$. (Conradia) 461

1218. P. seabra, B. \& $H$.

$$
\text { (Conradia) } 461
$$

1219. P. acaulis, B.\& $H$.

$$
\text { (C. Sloanei) } 462
$$

1220. P. Libanensis, $B . \& H$. (Conradia) 462

1221. P. pumila, B. \& $H$. (Conradia) 46:

\section{Rhytidophyllum}

1222. R. grande, Mart. 459

1223. R. tomentosum, Mart. 460

Tussacia

1224. T. pulchella, Reichent. 463

Drymonia

122.). D. parvifolia, Griseb. 463

Alloplectus

1226. A. repens, Hook.

(Pterygoloma) 464

1227. A. pubescens, $B$. \& $H$.

(Pterygoloma) 464

12:8. A. cristatum, B. i $H$.

(Pteryoroloma) 464

\section{Columnea,}

1299. C. argentea, Grisel. 465

139. C. hirsuta, Sw. 4 (tis

1231. C. hispida, $S(n .4(i)$

12:32. C. rutilans, sis. 416

Bisleria

1.:33. B. lutea, Lim. \pm 63 


\section{List of Jamaica Flowering Plants.}

\section{BigNoNIAOEE.}

\section{Pithecoctenium}

1234. P. Aubletii, Splitg. 449

Taniecium

1:35. T. Jaroba, Sw. 450

1236. T. exsertum, Grisel. 450

\section{Catalpa}

1237. C. longissima, šms. 446

Tecoma

12:38. T. Berterii, DC. 446

1239. T. leucoxylon, 11 (urt. 447

1240. T. pentaphylla, $D C .447$

1241. T. platyantha, Griseb. $4+7$

1242. T. stans, Juss. 447

Schlegelia

1243. S. parasitica, Moris. 115

\section{Creseentia}

1244. C. Cujete, Iinn. 445

1245. C. cueurbitina, Lim. 445

\section{Pedaline}

\section{Sesamum}

1246.*S. indicum, $D C$.

(S. orientale) 458

\section{ACANTHACE ZE.}

\section{Thunbergia}

1247.*T. fragrans, Roxb. 458

1248.*'T. alata, Bojer

\section{Ruellia}

1249. R. geminiflora, H.B.K. 451

1250. R. paniculata, Linn. 451

1251. R. tuberosa, Linn. 452

13.2. R. acuminata, Griseb. 452

1253. R. macroplyylla, Vahl (Stemonacanthus) 452 1:5t.*R. formosa, Ait.
Blechum

1255. B. Brownei, Juss 453

1256. B. laxiflorum, Juss. 453

Anthacanthus

12.57. A. armatus, Nees 457

12.58. A. jamaicensis, Griseb. 457

1259. A. microphyllus, Nees 457

Andrographis

1260.*A. paniculata, Nees 458

Geissomeria

1261. G. coccinea, T. Anders. 454

Beloperone

1.262. B. nemorosa, Nees 456

Dianthera

1263. D. seeunda, Griseb. 455

1264. D. pectoralis, II 4 rr. $45 \mathrm{~J}$

1265. D. reptans, Griseb. 455

1266. D. comata, Linn. 456

Jacobinia

1267. J. coccinea, B. \& $H$. (Pachystachys) 454

Thyrsacanthus

1268. 'T. nitidus, Nees 454

Dicliptera

1269. D. assurgens, Juss. 458

MYOPORINEE.

Bontia

1270. B. daphnoides, Linn. 503

VERBENACEE.

Lantana

1271. L. Camara, Linn. 495

1272. L. crocea, Jacq. 496

1273. L. involucrata, Linn. 496 (includes L. odorata) 496 
1274. L. reticulata, Pers. 496 1275. L. stricta, Sw. 496 1276. L. trifolia, Limn. 496

\section{Lippia}

1277. L. nodiflora, Michx. 494 1278. L. reptans, H.B.K. 495 1279. L. stœechadifolia, H.B.K. 495

Bouchea

1280. B. Ehrenbergii, Cham. 493

Stachytarpheta (Stachytarpha) 1281. S. cayennensis, Vahl 494 1282. S. jamaicensis, I'thl 494 1253." S. mutabilis, Vaht 494

Priva

1284. P. echinata, Juss. 493

\section{Verbena}

1285. V. urticefolia, Lim. 493 1286.*V. Bonariensis, Linn.

Citharexylum

1287. C. subserratum, Sw. 497 1288. C. quadrangulare, Jucq. 497 1289. C. cinereum, Linn. 497 1290. C. caudatum, Linn. 497 (includes C. lucidum) 497

1291. C. surrectum, Griseb. 497 1292. C. Berterii, Spreng. 498

\section{Duranta}

1293. D. Plumieri, Jacq. 498

\section{Callicarpa}

129t. C. ferruginea, $S w .499$

1:95. C. reticulata, $S w .499$

\section{Egiphila}

1296. As. trifida, $S w .499$

1297. Ze. fotidn, $S w .499$

1298. \#. martinicensis, Linn. 500 1299. 开. elata, Sw. 500

Petitia

1300. P. domingensis, Jacq. 501

\section{Cornutia}

1301. C. punctata, Willd. 501

Vitex

1302. V. umbrosa, Sw. 502

Clerodendron

1303. C. aculeatum, Griseb. 500

1304."C. fragrans, Vent. 500

Avicennia

1305. A. nitida, Jucq. 502

\section{LABIATAE.}

\section{Ocimum}

1306.* O. Basilicum, Linn. 487

1307. O. mieranthum, Willd. 487

\section{Coleus}

1308. ${ }^{* C}$. amboinicus, Lour. 487

Hyptis

1309. H. spicigera, Lam. 487

1310. H. capitata, Jacq. 488

1311. H. brevipes, Poit. 488

1312. H. atrorubens, Poit. 488

1313. H. spicata, Poit. 488

1314. H. suaveolens, Poit. 489

1315. H. pectinata, Poit. 489

1316. H. verticillata, Jacq. 489

Micromeria

1317. M. obovata, Benth. 489

1318. M. Brownei, Lenth. 48 ?

Salvia

1319. S. oecidentalis, ste. t90

1320. S. mier:untha, Vahl (S. tenella) 490

1:321. A. serotinil, Lim. 490

1322. S. hispanica, Lim. 490

1:3)?. S. eriocalyx, Berter. 490

1:3-t. A. coccinea, Lmm. 490

Brunella (Prunctla)

1305. B. vulgaris, Lim. 492 


\section{List of Jamaica Flowerng Plants.}

Anisomeles

1326.*A. ovata, R. Br. 491

Stachys

1327.*S. arvensis, Linn. 491

\section{Leonurus}

1328." L. sibiricus, Linn. 491

\section{Leonotis}

1329.*L. nepetæefolia, R. Br. 492
Teuerium

1330. T. inflatum, Sw. 492

\section{Plantaginem.}

\section{Plantago}

1331. P. major, Linn. 389

1332. P. virginica, Linn. 389

1333.*P. lanceolata, Linn.

\section{Division III.-MONOCHLAMYDEÆ.}

\section{NYOTAGINE君.}

\section{Mirabilis}

1334. M. Jalapa, Linn. 69

\section{Boerhaavia}

1335. B. erecta, Linn. 69

1336. B. paniculata, Rich. 69

1337. B. hirsuta, Willd. 69

1338. B. seandens, Linn. 69

\section{Pisonia}

1339. P. aculeata, Linn. 70

1340. P. nigricans, Sw. 70

1341. P. inermis, Jacq. 71

1342. P. obtusa, Swo 71

\section{Neea}

1343. N. jamaicensis, Griseb. 71

\section{AMARANTACE}

\section{Celosia}

1344. C. argentea, Linn. 62

1345. C. nitida, Vahl 62

\section{Chamissoa}

1346. C. altissima, H. B. K. 62

Amarantus

1347. A. spinosus, Linu. 68

1318. A. tristis, Linn. 69
1349. A. polygonoides, Linn. (Amblogyne) 68

1350. A. crassipes Schlecht. (Scleropus) 68

1351. A. Blitum, Linn. (Euxolus viridis) 68

1352. A. viridis, Linn. (Euxolus caudatus) 68

Acnida

1353. A. cannabina, Linn. 60

Cyathula

1354. C. prostrata, Blume 63

Achyranthes

1355. A. aspera, Linn. 62

Alternanthera

1356. A. polygonoides, R. Br. 67

1357. A. ficoidea, $R . B r .67$

Philoxerus

1358. P. vermicularis, R. $B r .65$

Gomphrena

1359. G. globosa, Linn. 63

Frolichia

1360. F. interrupta, Moq. 63

Iresine

1361. I. celosioides, Linn. 64 1362. I. elatior, Kich. 61 


\section{Chenopodiacez.}

Chenopodium

1363. C. ambrosioides, Linn. 60

Anredera

1364. A. scandens, Moq. 60

\section{Phytolaccace⿸.}

Rivina

1365. R. humilis, Livm. 59

Villamilla

1366. V. octandra, Hook. $f$. (Rivina) 59

Petiveria

1367. P. alliacea, Linn. 59

Phytolatera

1368. P. octandra, Linn. 58

1369. P. icosandra, Linn. 58

Batis

\section{BATIDEÆ.}

1370. B. maritima, Linn. 61

\section{POLYgONACE 灰.}

Polygonum

1371. P. acre, H. B. K. 161

1372. P. acuminatum, H.B.K. 161

1373. P. glabrum, Willd. 161

\section{Coccoloba}

1374. C. uvifera, Jacq. 161

1375. C. leoganensis, Jacq. 162

1376. C. tenuifolia, Linn, 1(i)

1377. C. Plumieri, Griseb. 10:

1378. C. Zebra, Griseb. 16:

1379. C. diversifolia, Jacq. 163

1380. C. punctata, Linn. 163

1381. C. nivea, Jnc\%. 163

\section{ARISTOLOCHIACE 丑.}

Aristolochia

1382. A. ringens, $V a h l 299$

1383. A. grandiflora, Sw. 299

1384. A. odoratissima, Linn. 299

1385. A. trilobata, Linn. 299

\section{Piperace..}

Piper

1386. P medium, Jacq.

(Enkea Swartzii, \&

E. Sieberi) 169, 170

1387. P. ceanothifolium, H.B.K. (E. Amelago, v. hirtella) 169

1388. P. Amelago, Linn.

1389. P. Berteroanum, C. DC. 1390. P. Wulfsehlægelii, C. DC. 1391. P. unguiculatum, Ruiz.\& Pav.

(E. Amelago) 169

1392. P. panduratum, C. $D C$.

1393. P. genieulatum, Sw.

(Artanthe geniculata, verucosa, ma.crophylla, \& jamaicensis) 170-2

1394. P. hirsutum, Sw. (A scabra) 171

1395. P. Murrayanum, C. DC? 1396. P. Fadyenii, C. $D C$. 1397. P, aduncum, Linn. (A. adunca) 171

1398. P. reticulatum, Sw. 1399. P. dilatatum, Rich. 1400. P. peltatum, Linn. (Potomorphe) 169

1401. P. umbellatum, Linn. (Potomorphe) 169

1402. P. laueifolium, Hari.

Verhuellia

1403, V. serpens, MLir. (Pepromia) 164

Peperomia 1404. P. tenella, A. Dietr. 165 
1405. P. hispidula, A. Dietr. 165 1406. P. exilis, Griseb. 164 1407. P. pellucida, H.B.K. 165 1408. P. melanostigma, Mir. 1409. P. dendrophila, Schlecht. 165 1410. P. nemorosa, C.DC. (P. acuminata) 165

1411. P. glabella, A. Dietr. 1412. P. nummularifolia, H.B.K. 164

(includes P. rotundifolia) 164 1413. P. cordifolia, A. Dietr. 164 1414. P. Hamiltoniana, Miq. 1415. P. amplexicaulis, A. Dietr. 1416. P. amplexifolia, A. Dietr. 1417. P. magnoliæfolia, C.DC. 1418. P. alpina, A. Dietr. 167 1419. P. producta, Griseb. 166 1+20. P. scandens, Ruiz \& I'ar. (P. repens) 165

1421. P. coucinna, A. Dietr. 1422. P. septemnervis, Ruiz \& Pav. 168

1423. P. Myrtillus, Miq. 168 1424. P. reflexa, A. Dictr. 168 1425. P. stellata, A. Dirtr. 167 1426. P. quadrifolia, H.B.K. 168 1427. P. pulchella, A. Dietr. 167 (inchudes P. verticillata) 167 14:25. P. discolor, C. DC. 1429. P. galiodes, H.B.K. 167 1430. P. Swartziana, Miq. 167

\section{LAURINE鹿.}

Beilschmiedia

1431. B. Thomea, B. \& H. 1432. B. pendula, $B$. \& $H$. (Hufelandia) 280

\section{Acrodiclidium}

1433. A. Jamaicense, Nees. 280
Cinnamomum

1434.*C. zeylanicum, Breyn. 279

1435. 'C. Camphora, Nees. \& Eberm.

Persea

1436. P. gratissima, Gertn. 280

1437. P. alpigena, Spreng. 280

1438. P. montana, Spreng.

$$
\text { (Phobe) } 279
$$

1439. P. staminea, $B . \& H$. (Nectandra) 282

Ocotea

1+40. O. botryophylla, Klotsch. \& Karst.

(Nitychnodaphne floribunda) 283.

1411. O. Leueoxylon, $B . \& H$. (1)reodaphne) 283

1412. 0. parviflora, $B . \& H$. (Oreodaphne) 283

Nectandra

1443. N. Antillana, Meissn. (N. leucantha) 282

1444. N. membranacea, Griseb. 282

1445. N. coriacea, Griseb. 281 14ti. N. patens, Griseb. 281

147. N. Willdenoviana, Nees. (N. sanguinea, partly) 281

148 N. exaltata, Griseb. 281

Cassytha

1449. C. americana, Nees. 285

Hernandia

1450. H. sonora, Limn. 285

Thyameleacee.

Daphnopsis

14.5l. D. Swartzii, Meissn. 278 1452. D. tinifolia, Griseb. 278 


\section{Lagetta}

1453. L. lintearia, Lam. 279

\section{LORANTHACEE.}

\section{Loranthus}

1454. L. claviceps, Griseb. 311 1455. L. lepidobotrys, Griseb. 311 1456. L. avicularius, Mart. 312 1457. L. parvifolius, Sw. 312 1458. L. uniflorus, Jacq. 312 1459. L. emarginatus, $S w, 312$ 1460. L. pauciflorus, $S w .312$ 1461. L. occidentalis, Linn. 313

Arceuthobium

1462. A. cupressoides, Griseb. 315 1463. A. gracile, Griseb. 315 1464. A. opuntioides, Griseb. 315

Phoradendron

1465. P. flavens, Griseb. 313

1466. P. Berterianum, Griseb. 313 1467. P. latifolium, Griseb. 314 1468. P. rubrum, Griseb. 314 1469. P. trinervium, Griseb. 314

\section{BALANOPHORER.}

Serbalium

1470. 's. jamaicense, Eichl. (Phyllocoryne) 309

\section{EUPHORBIACEEA.}

Pedilanthus

1471. P. angustifolius, Poit. 52

\section{Euphorbia}

1472. E. alata, Hook. 52

1473. E. nudiflora, Jacq. 52

1474. E. myrtillifolia, Limn.

(E. myrtifolia) 53.
1475. E. buxifolia, Lam. 53 1476. E. thymifolia, Burm. (E. maculata) 53

1477. E. prostrata, Ait. 53 1478. E. pilulifera, Linn. 54 1479. E. hypericifolia, Linn. 54 1480. E. Brasiliensis, Lam. (E. hyssopifolia) 54 1481. E. lasiocarpa, $K l .54$ 1482. E. punicea, $S w .54$ 1483. E. heterophylla, Lim. 54 1484. E. geniculata. Ort. (E. prunifolia) 54 1485.*E. Peplus, Linn. 54

Buxus (Tricera)

1486. B. lævigata, Spreng. 31 1487. B. Purdiana, Brill. (T. fasciculata) 31

1488. B. Vahlii, Buill.

Phyllanthus

1489. P. distichus, Muell. Arg. (Cicca disticha) 32

1490. P. nobilis, Muell. Arg. (C. antillana) 33

1491. P. Epistylium, Griseb. 33

1492. P. cauliflorus, Griseb. 33

1493. P. nutans, Sw. 33

1494. P. Conami, su. 33

149.. P. Niruri, Lim. 34

1496. P. tremulus, Griseb. 34

1497. $P$. reticulatus, $P$ oir.

(P. jamaicensis) 34

1498. P. orbicularis, H. B. K. 34

1499. P. montauus, $S w .35$

1500. P. Epiphyllanthus, Muell. Arg.

(P. falcatus) 35

1501. P. angustifolius, Sw. 35

1502. P. linearis, Sw. 35

1503. P. latifolius, Swo. 35

1504. P. speciosus, Jacq. 35

\section{Drypetes}

1.305. D. crocera, Poit. 32 


\section{- List of Jamaica Flowering Plants.}

1506. D. glauca, Vahl. 32

\section{Hieronyma}

1507. H. alchorneoides, Allem. 32

Jatropha

1508. J. gossypifolia, Linn. 36

1509.*J. multifida, Jinn. $3 b^{3}$

1510. J. divaricata, $S w .36$

1511. J. hernandæfolia, Vent. 36

1512. J. Curcas, Limn. 36

\section{Aleurites}

1513. A. moluccana, Willd.
(A. triloba) 37

\section{Croton}

1514. C. linearis, Jacq.

(C. Cascarilla) is

1515. C. flavens, Lim 38

(includes $v$. balsamifer) 38

1516. C. corylifolius, Lam. 39

1517. C. Grisebachianus, Muell. Arg. (C. Iserti) 39

1518. C. glabellus, Muell. Arg.

(C. Eluteria) 39

1519. C. Laurinus, Sw. 39

1520. C. micans, Sic.

(C. niveus) 40

1521. C. lucidus, Linn. 40

15.2. C. Wilsonii, Griseb. 40

1523. C. adenophyllus, Spreng. 40

1524. C. humilis, Linn. 41

1525. C. ovalifolius, West 41

1526. C. populifolius, Jinn. 41 (includes $\mathrm{v}$. hirtus) 42

\section{Argithamnia}

1527. A. candicans, $S t \cdot .44$

\section{Caperonia}

1528. C. palustris, Sw. Hil. 43

\section{Manihot (Janipha)}

1529.*M. utilissima, Pohl. 37

1530.*M. Aipi, Pohl. 37
Bernardia

1531. B. carpinifolia, Griseb. 45

1532. B. Corensis, Muell. Arg. (Polyboea) 46

Acalypha

1533. A. scabrosa, $S w .47$

(includes v. betulæfolia) 47

1534. A. virgata, Linn. 47

1535. A. elliptica, $S w .47$

1536. A. hernandifolia, $S w .47$

1537. A. villosa, Jacq. 47

1538. A. lævigata, $S w .48$

1539. A. cuspidata, Jacq. 48

1540. A. chamædrifolia, Iuell. Arg. (A. reptans) 48

Adelia

1541. A. Ricinella, Lim. 4.5

1542. A. hæmiolandra, $B . d^{*} H$. (Ditaxis) $4+$

\section{Alchornea}

1543. A. latifolia, $S w .46$

\section{Lasiocroton}

1544. L. macrophyllus, Griseb. 46

Ricinus

1545. R. communis, Lins. 37

Mettenia

1546. M. globosa, Griseb. 43

Acidoton

1547. A. urens, $S w .45$

Tragia

1548. T. volubilis, Linn. 48

Omphalea

1549. O. triandra, Linn. 50

1550. O. diandra, Linn. 50

\section{Hippomane}

1551. A. Maneinella, Linn. 50

Sapium

1552. S. Laurocerasus, Muell. Arg. (S. laurifolium) 49 
1553. S. cuneatum, Griseb. 49

Gymnanthes (Excoecaria)

1554. G. Iucida; Sw. 50

1555. G. elliptica, Sw.

$$
\text { (E. tinifolia) } 51
$$

1556. G. glandulosa, Muell.

$$
\text { Arg. } 51
$$

\section{Hura}

1557. H. crepitans, Linn. 50

\section{URTICACE AT.}

(eltis

1558. C. Jamaicensis, Planch.

1559. C. trinervia, Lam. 149

1560. C. aculeata, Sw. 149

Trema (Sponia)

1561. T. mierantha, B. \& H. 150

1562. T. Lamarckiana, $B . \&$ \& $H .150$

Cannabis

1563."C. sativa, Linn.

Chlorophora

1564. C. tinctoria, Gaud. (Maclura) 153

\section{Trophis}

1565. T. americana, Linn. 1.53

\section{Dorstenia}

1566. D. cordifolia, Lam. 154

\section{Ficus}

1567. F. laurifolia, Lam. 150

1568. F. suffocans, Hb. Banks 150

1569. F. crassinervia, Desf. 150

1570. F. trigonata, Limn. 150

1571. F. lævigata, Vahl 151

1572. F. dimidiata, Griseb. 151

1573. F. ochroleuca, Grisel. 151

1574. F. pedunculata, Ait. 151

1575. F. pallida, Vahl 151

157 (j. F. pertusa, Linn. 15?
Brosimuum

1577. B. Alicastrum, Sw. 152

Pseudolmedia

1578. P. spuria, Griseb. 152

Artocarpus

1579.*A. incisa, Linn. 152

1580.*A. integrifolia, Linn. 152

Cecropia

1581. C. peltata, Linn. 153

Fleurya

1582. F. æstuans, Gaud. 154

Urera

1583. U. caracasana, Gruetrl. 154

1584. U. elata, Griseb. $15 t$

1585. U. expansa, Griseb. 155

1586. U. baceifera, Grutl. 155

1587. U. microcarpa, Wedd. 155

Gyrotænia

1588. G. spicata, Wedd.

(Urera, spicata) 155

Pilea

1589. P. mierophylla, Liebm. 155

1590. P. trianthemoides, Lindl. 155

1591. P. lanceolata, Wedd. 156

150.2. P. nudieanlis, Tredd. 1.56

1593. P. virgata, Wedr.

1594. P. ciliaris, Wedt. 156

(includes P. Parietaria) 156

1595. P. lucida, Bl. 156

1596. P. cuneifolia, Wedd. 156

1597. P. clandestina, Tredd.

1598. P. rufa, Wedd. 157

1599. P. serrulata, Wedd. 156

1600. P. grandis, Well. 157

1601. P. crassifolia, $B$.

(P. densiflora) 157

1602. P. reticulata, Wedd. 156

1603. P. sessiliflora, Wrdl. 157

1604. P. radicans, Wedr. 158

160.). P. inæqualis, Wedd. 158 
1606. P. pubescens, Liebm. 159 1607. P. diffusa, Wedd. 159 160S. P. laxa, Wedd. 159 1609. P. repens, Wedd. 158 1610. P. rotundata, Griseb. 158 1611. P. nummularifolia, Wedd.1.5s 1612. P. depressa, Bl. 1.58 1613. P. swartzii, Wedd. 158

\section{Bohmeria}

1614. B. caudata, $S w .160$

1615. B. cylindrica, Wintd. 160

1616. B. ramiflora, Jacq. 160

\section{Phenax}

1617. P. hirtus, Wedt.

(P. urticifolius) 160

Rousselia

1618. R. lappulacea, Gamd. 160

MrRiché.

Myrica

1619. M. microcarpa, Benth. 177
1620. M. microstachya, $K r$. \& Urb.

\section{Casuarinez.}

('asuarina

1621.*C. equisetifolia, Forst. 177

\section{SaLICINEe.}

Salix

16.2. S. Humboldtiana, Witld. 113

La'ISTEMAC'E.E.

Lacistema

162:?. L. myricoides, Su, 25

Ceratophylle.

Ceratophyllum

1624. C. demersum, Lim. 12

\section{Class II.-MONOCOTYLEDONES.}

\section{SERIES I-MICROSPERMIAE.}

\section{HIDROCHARDEE正。}

Thalassia

16:- T. testudinum, Kœn. 507

\section{BURMANNIACEE.}

\section{Burmannia}

1626. B. capitata, Trort. 606

\section{Apteria}

10i27. A. setacea, Nutt. 606

\section{ORCHIDEE.}

Pleurothallis

1628. P. univaginata, Lindl. 607 1629. P. gelida, Lindl. 607 1630. P. alpestris, Lindl. 607 1631. P. laxa, Lindl. 607 1632. P. longissima, Lindl. 607 1633. P. racemiflora, Lindl. 607 1634. P. Wilsoni, Lindl. 608 16i35. P. ruscifolia, $R . B r .608^{\circ}$ 1636. P. pruinosa, Lindl. 608 
1637. P. emarginata, Lindl. 608 1638. P. delicatula, Lindl, 608 1639. P. corniculata, Lindl. 609 1940. P. lanceola, Spreng. 609 1611. P. tribuloides, Lindl. 609 16t:. . P. sertularioides, Spreng. 609 1643. P. testifolia, Iindl. 609 1644. P. foliata, Griseb. 610 164.5. P. microlepanthes, Griseb. 610

Stelis

1646. S. ophioglossoides, Sw. 611 16t7. . . micrantha, s'u. 611

Lepenthes

1648. L. rotundata, Griseb. 610 1649. L. tridentata, Sw. 610 1650. L. pulchella, $S w .610$ 16.1. L. sanguinea, Hook. 611 1652. L. concinna, Swo. 611 16is. L. cochlearifolia, Sw. 611

Masidevallia

1654. M. fenestrata, Iindl. 612

Arpophyllum

1655. A. spicatum, Llav \& Lex.

Oetomeria

1656. O. graminifolia, R. Br.611

Microstylis

1657. M. umbellulata, Liudl. 61'] 1658. M. spicata, Lindl. 612

\section{Liparis}

16.59. L. elliptica, Reichenb. f. 612 1660. L. elata, Linn. 612

Bulbophyllum

1661. B. pachyrrachis, Griseb. 613 Coelia

1662. C. triptera, Don. 629

Phaius

1663.*P. grandifolius, Lour. 622
Bletia

1664. B. florida, $R$. $B r .621$

1665. B. verecunda $R . B r .622$

1666. B. Shepherdii, Hook. 622

Calanthe

1667. C. mexicana, Reichenb. $f$.

Elleanthus (Evelyna)

1668. E. capitatus, Reichenb.f. 622 1669. E. longil)rateatus, 'Benth. 623

\section{Hormidium}

1670. II. pyemixum, Isruth. (Epidendrum) 615

Octadesmia

1671. O. montana, isenth. (Tetramicra montana) ( 022 1672. O. elata, Benth. 1673. O. monophylla, Benth. (Trigonidium) 629

\section{Isochilus}

1674. I. linearis, R.Br. $6 \mathrm{w}^{3}$

Ponera

1675. P. prolifera, Reichent. f. 69:?

Epidendrum

1676. E. angustifolium, Sw. 613

1677. F. polybulbon, Sw. 615

1678. E. bletioides, Griseb. 615

1679. E. serrulatum, Reichenb.f. 615

1680. E. ciliare, Limn. 615

1681. E. fragrans, $S w .615$

1682. E. cocbleatum, Lim. 616

1683. E. spondiadum, Reichenb. $f$.

1684. E. rivulare, Limll. 616

1685. E. discoidale, Lincll. 616

1686. E. nutans, s'r. 616

1687. E. fuscatum, $S w .1317$

1688. E. jamainensw', Limdl. 618

1689. E. bifarium, $S w .618$

1690. E. rigidum, Jor't. 618 


\section{List of Jamaica Flowering Plants.}

1691. E. strobiliferum, Reichenb.f. 618.

1692. E. ramosum, Jacq. 618 1693. E. umbellatum, $S w .618$ 1694. E. nocturnum, Linn. 619 1695. E. diffusum, $S w \cdot 619$ 1696. E. verrucosum, $S w .619$ 1697. E. stenopetalum, Hook. 619 1698. E. teretifolium, $S w$. fil9 1699. E. globosum, Jacq. 619

Broughtonia

1700. B. sanguinea, R. $\mathrm{Br} .620$

1701. B. lilacina, Henfr.

(Læliopsis) (i:1

Tetramicra

1702. T. rigida, Lindl. 62:2

1703. T. parviflora, Lindl. 622

Brassavola

1704. B. cordata, Lindl. 6:0

1705. B. Sloanei, Griseb. 620

1706. B. nodosa, Lindl. 621

1707. B. vomeriformis, Reichenb. $f$. $6: 1$

Schumburgkia

1708. S. Lyonsii, Lindl. (s. carinata) (jol

Polystachya

1709. P. luteola, Hook. 628

1710. P. foliosa, Reichenb. $f .628$

Cyrtopodium

1711. C. Woodfordii, Benth. (Cyrtopera) 630

Govenia

1712. G. utriculata, Lindl. 628

Zypopetalum

1713. Z. cochleare, Lindl. 629

Xylobium

1714. X. palmifolium, Benth. (Maxillaria) 627
1715. X. pallidiflorum, Benth

(Maxillaria) 627

\section{Lycaste}

1716. L. Barringtoniæ, Lindl. 627

Stenia

1717. s. pallida, Lindl. 629

Maxillaria

1718. M. alba, Lindl. 626

1719. M. crassifolia, Reichenb.f. 626

Dichæa

1720. D. echinocarpa, Lindl. 624

1721. D. muricata, Liudl. 624

1722. N. trichocarpa, Lindl. 624

172:3. D. graminea, Griseb. 625

17:4. D. glauca, Limdl. 625

Ornithidium

1725. O. vestitum, I'eichenb. $f .625$

17:6. O. confertum, Griseb. 626

1727. O. coccineum, Salisb. 626

Comparettia

1728. C. falcata, Pcpp.\& Endl. 636

Odontoglossum

17.9. O. jamaicense, Griseb. 633

Oncidium

1730. O. 1riquetrum, $R . B r .631$

1731. O. pulchellum, Hook. 631

1732. O. tetrapetalum, Willd. 631

1733. O. variegatum, $S w .632$

1734. O. sylvestre, Lindl. 632

1735. O. luridum, Lindl. 632

1736. O. altissimum, Sw. 632

Brassia

1737. B. maculata, R. Br. 633

1738. B. caudata, Lindl. 633

Leiochilus

1739. L. cochlearis, Jindl. 634

Ionopsis

I740. I. testiculata, Lindl. 636 
1741. I. utricularioides, Lindl. 636

Cryptarrhena

1742. C. lunata, $R . B r .635$

Dendrophylax

1743. D. filiformis, Benth.

$$
\text { (Aeranthes) } 625
$$

1744. D. funalis, Benth.

$$
\text { (Aeranthes) (j:5) }
$$

\section{Campylocentron}

1745. C. jamaicense, Benth.

(Aeranthes micrantha) 625

Macradenia

1746. M. lutescens, $R . B r .635$

Vanilla

1747. V. anaromatica, Griseb. 638 1748. V. claviculata, Sw. 638

Corymbis (Chloidia)

1749. C. flava, Hemsl. 643

1750. C. polystachya, Benth. 643

Cranichis

1751. C. muscosa, $S w .639$

1752. C. pauciffora, $S w .639$

1753. C. ventricosa, Griseb. 639

1754. C. diphylla, $S w .639$

\section{Prescottia}

1755. P. stachyoides, Lindl. 639

1756. P. myosurus, Reichenb.f. 640

Ponthieva

1757. P. glandulosa, $R . B r .638$

1758. P. petiolata, Lindl. 638

Wullschlægelia

1759. W. aphylla, Reichenb. f. 639

\section{Pseudocentrum}

1760. P. minus, Benth.

\section{Stenoptera}

1761. S. ananassocomos, Reichenb.f. 640

\section{Spiranthes}

1762. S. tortilis, Rich. 640

1763. S. apiculata, Lindl. 640

1764. S. elata, Rirh. 641

1765. S. speciosus, Benth . (Stenorrhyneus) $6 \pm 2$

1766. S. lanceolatus, Benth. 642

1767. S. orchioides, Hemsl. 642

1768. S. tenue, Benth.

$$
\text { (Sauroglossum) } 612
$$

1769. S. adnata, Benth.

(Pelexia spiranthoides) $6 \pm 2$

\section{Pelexia}

1770. P. setacea, Lindl. 642

Physurus

1771. P. hirtellus, Lindl. 642

1772. P. plantagineus, Lindl. 643

Pogonia

1773. P. physurifolia, Reichenb. $f$. 637

1774. P. gentianoides, Lindl. 637

Habenaria

1775. H. macroceratitis, Willd. 643

1776. H. maculosa, Lindl. 643 1777. H. alata, Hook. $64 t$

\section{SERIES II.-EPIGYNAF.}

SCITAMTNEA.

Hedychium

1778.*H. Gardnerianum, Rose.

\section{Zingiber}

1779.*Z. officinale, Rose. 602

Costus

1780. C. spicatus, $S w .602$ 


\section{List of Jamaica Flowering Plants.}

Renealmia

1781. R. sylvestris, Griseb. 601

1782. R. occidentalis, Griseb. 601

1783. R. racemosa, Rosc. 601

\section{Maranta}

1784. M. arundinacea, Limn. 605

\section{Canna}

1785. C. indica, Limn. 603

1786. C. coccinea, Ait. 603

1787. C. pallida, Rose. 603

1788. C. glauca, Limn. 603

Heliconia

1789. H. Bihai, Linn. 600

1790. H. psittacorum, Linn. 600

\section{Musa}

1791.*M. paradisiaca, Linn. 599 (includes v. sapientum) 599

BROMELIACE,

Karatas (Nidularium)

1792. K. Plumieri, E. Morr. 591

\section{Ananas}

1793. A. sativus, Lindl. 591

Bromelia

1791. B. Pinguin, Linn. 591

\section{Edchmea}

1795. Æ. paniculigera, Griseb. 592 1796. 对. lingulata, Bak.

(Chevalliera) 591

1797. Ae. polycephala, Bak.

1798. 2. aquilega, Griseb. 592

1799. 玵. distans, Griseb. 592

1800. Æ. bracteata, Griseb 592.

\section{Pitcairnia}

1801. P. bromeliæfolia, L'Her. 594

\section{Caraguata}

1802. C. lingulata, Lindl. 598
1803. C. Sintenesii, Bak.

Guzmania

•1804. G. tricolor, Ruiz \& Pav. 598

Catopsis

1805. C. nitida, Bak.

(C. nutans) 599

1806. C. nutans, Bak.

(C. nitida) 599

Tillandsia

1807. T. usneoides, Linn. 598 1808. T. recurvata. Linn. 598 1809. 'T. pruinosa, Sw. 596 1810. T. compressa, Bert. 595 1811. T. bulbosa, Hook. 596. 1812. T. canescens, sw. 595 1813. T. setacea, Sw. 595 1814. 'T'. angustifolia, Sw. 595 1815. T. Balbisiana, Schult. 595 1816. T. fasciculata, Sw. 595 1817. T. sublaxa, Bak.

1818. T. valenzuelana, A. Rich. (T. laxa) 596

1819. T. flexuosa. Sw. 596

1820. T. Swartzii, Bak.

1821. T. complanata, Benth. ( $\mathrm{T}$ axillaris) 597

1822. T. excel $\$$, Griseb. 597

HAEMODORACEA.

Xiphidium

1823. X. floribundum, Swo. 589

IRIDEAE.

Moræa

1824.* M. iridioides, Linn.

Marica

1825.*M. cœrulea, Ker. 
Trimezia

1826. T. martinicensis, $B$. \& $H$. (Cipura) 589

Tigridia

1827.*T. Pavonia, Ker.

Eleutherine

1828. E. plicata, Herb.

Belamcanda

(Cipura) 589

1829. 'B. chinensis, Leman.

\section{AMARYLLIDE.E.}

Hypoxis

1830. H. decumbens, Linn, is.5

Cureuligo

1831. C. scorzoneræfolia, Bentte. (Hypoxis) 585

Zephyranthes

1832. Z. tubispatha, Herb.

$$
\text { (Amaryllis) } 584
$$

1833. Z. carinata, Herb. 584

\section{Hippeastrum}

1834. H, equestre, Herb.

(Amaryllis) 584

Crinum

1835. C. caribæum, Bak.

(C. floridanum) 583
1836. C. erubescens, Soland. 583 1837.*C. giganteum, $A n d r .584$ 1838. C. longiflorum, Herb. 583

Hymenocallis (Pancratium)

1839. H. caribæa, Herb. 583

1840. H. caymanensis, Herb. (P. patens) 583

Agave

1841. A. Morrisii, Bak.

(A. americana) 582

Furcroea

184.. F. eubensis, Haw. 582

\section{Dioscoreace.a.}

\section{Dioscorea}

1843. D. cayennensis, Lam. 187

1844.* D. bulbifera, Linn. 587

1845.*D. alata, Limn. 587

1846.' D. truphylla, Iimm. 587

1847.*D. trifida, Lim 587

1848. D. pilosiuscula, Berter. 588

1849. D. multiflora, Presl 588

Rajania

18.50. R. ovata, Sio. 588

\section{SERIES III.--CORONARIEAE.}

LILIACE 疋.

Smilax

1851. S. subaculeata, Spreng. 586

1852. S. Balbisiana, Kunth. 586

1853. S. celastroides, Kunth. 586

1854. S. viseifolia, Poir. 586

Hemerocallis

1855*H. fulva, Timn.
Aloe

1856. A. vulgaris, Limn. 582

Yucca

18.7. Y. aloifolia, Lim. 582

Agapanthus

1858. ${ }^{*}$ A umbellatus, $L^{\prime} H e r$.

Allium

1859. A. striatum, Jac\%. 581 


\section{List of Jamaica Flowering Plants.}

Pontederiace.

Heteranthera 1860. H. limosa, Vahl 590

Eichornia

1861. E. paniculata, $K$. Spreng.

(E. tricolor) 590

1862. E. azurea, Kunth 590

\section{MaYACE正.}

Mayaca

1863. M. fluviatilis, Aubl. 526

Commelinacee.

Commelina

1864. C. nudiflora, Lim.

(C. carennensis) 524
1865. C. Virginica, Linn. (C. elegans) 525

Tinantia

1866. T. fugax, Scheidw.

Tradescantia

1867. T. cordifolia, Str.

1868. T. multiflora, Sw.

Campelia

1869. C. Zanonia, H. B. $K .523$

Rhæo

1870. R. discolor, Hance

(Tradescantia) 524

Zebrina

1871.*Z. pendula, Schnizl.

SERIES IV.-CALTCINLE.

JUACACE.

Juneus

18:2. J. tenuis, Willd. 581

PAIM.E.

Euterpe

1873, E. oleracea, Mart. 517

Oreodoxa

1s.t. O. oleracea, Mart. .517

Calyptrogyne (Calyptronoma) 1875. C. Swartzii, H. Wendl. 518

Sabal

1876. S. umbraculifera, Mart. $51 t$

Copernicia

1877. C. tectorum, Mart. 514

\section{Thrinax}

18.8. T. parviflora, Sw. 515

1879. T. argentea, Lodd. 515

1880. T. excelsa, Zodd. 515

Bactris

1881. B. Plumieriana, Mart. 520

Acrocomia

1S82. A. selerocarpa, .Mait. 521

1883. A. lasiospatha, Mart. 521

Elæus

1884."E. guineensis, Linn. 522

Cocos

1885. C. nueitera, Linn. 522

1886. C. amara, Jacq.

(Syagrus) 522

\section{SERIES T.-NLDIFLOREE.}

CrCLANTHACEE.

Carludovica

1597. C. Plumieri, Kunth 513
Trphace.

Typha

1888. T. angustifolia, Limn. 512 
AROIDEÆ.

Anthurium

1889. A. scandens, $\boldsymbol{A} u b l$. (A. violaceum) 507

1890. A. Hookeri, Kunth

$$
\text { (A. Huegelii) } 508
$$

1891. A. cordifolium, Kunth 508

\section{Syngonium}

1892. S. auritum, Linn. 511

Philodendron

1893. P. lingulatum, Schott 510

1894. P. lacerum, Schott 510

Dieffenbachia

1895. D. Seguine, Schott 509

\section{Xanthosoma}

1896. X. sagittifolium, Schott 511

1897. X. atrovirens, C. Koch \& Bouch. 511

\section{Colacasia}

1898. C. Antiquorum, Schott 511

Pistia

1899. P. Stratiotes, Linn. 512

\section{LEMNACE 现.}

Wolffia

1900. W. punctata, Griseb. 512

\section{Stries VI.-APOCLIPPE.}

\section{ALISMACE巴.}

Echinodorus

1901. E. rostratus, Engelm.

(E. cordifolius) 505

\section{Sagittaria}

1902. S. lancifolia, Linn. 505

1903. S. montevidensis, Cham. of schlecht.
1904. S. intermedia, Mich. (S. acutifolia) 505

\section{NaIADACE F.}

\section{Potamogeton}

1905. P. fluitans, Roth. 506

1906. P. plantagineus, $D_{u}$ Croz 506

1907. P. pauciflorus, Pursh.

\section{SerIEs VII.-GLT $M A C E A E$.}

\section{CYPERACE涩.}

\section{Cyperus}

1908. C. polystachyus, Rottb. 562

1909. C. melanostachyus, H.B.K. (C. variegatus) 562

1910. C. lævigatus, Liun. (C. mucronatus) 562

1911. C. compressus, Lim. 562 1912. C. acuminatus, Tor $\% 563$ 1913. C. temuis, Sw. .j3

1914. C. confertus, $S 20.563$

1915. C orbraceus, I'ah 563
1916. C. viscosus, Ait. 563

1917. C. elegans, Linn. (C. laxus,) 563

1918. C. Haspan, Linn. 564 1919. C. articulatus, Linn, 564 1920. C. trichodes, Griseb. 564 1!'1. ('. rotundus, Limn. 564 1!'.2.) C. esculentus, Iinn. 565 1923. ('. brunneus, Siv. 565 1921. C. sphacelatus, Rottb. 565 1925. C. distans, Limn. f. 565 1926. C. filiformis, Sw. 565 1!27. (. odoratus, Limm. 565 
1928. C. densiflorus, Mey. 566

1929. C. flexuosus, Vahl 566

1930. C. Vahlii, Stend. 566

1931. C. Ehrenhergii, Kunth 566

1982. C. elatus, Linn. 566

1933. C. ligularis, Linn. 566

1934. C. Meyenianus, Kunth 567

1935. C. anceps, Liebm. 567

1936. C. Mutisii, Griseb. 567

1937. C. platystachyus, Griseb. 567

1938. C. cyclostachyus, Griseb. 567

1939. C. flavus, Boeckl.

(C. flavamariscus) 567

1940. C. Hartii, Boeckl.

1941. C. Swartzii, Boeckl.

(Kyllinga filiformis) 568

1942. C. umbeliatus, Benth.

\section{Kyllinga}

1943. K. triceps, Rottb. 568

194. K. monocephala, Rottb. 568

1945. K. brevifolia, Rottb. 568

1946. K. vaginata, Lam.

$$
\text { (K. aphylla) } 568
$$

1947. K. pumila, Michx.

\section{Heleocharis}

1948. H. chætaria, Roem. Schult (Scirpus retroflexus) 569

1949. H. ochreatus, Steud. 570

1950. H. capitata $R . B r .570$

1951. H. geniculata, Roem. \& Schult,

(S. constrictus) 570

1952. H. interstincta, Roem. \&

Schult. (S. plantagineus) 571

1953. H. mutata, Roem. \& Schult.

571

\section{Dichromena}

1954. D. pusilla, Kunth

(Rhyncospora) 576

1955. D. leucocephala, Michx.

(R. stellata) 576
1956. D. nervosa, Vahl

(R. pura, \& R. Vahliana) 577

1957. D. Persooniana, Nees 577

Fimbristylis

1958. F. autumnalis, Roem, \& Schult. (Scirpus) 571

1959. E. ferruginea, Vahl 572

1960. F. spadicca, Vahl 572

1961. F. polymorpha, Boeckl.

(S. brizoides) 572

1962. F monostachya, Hasst.

(Abildgaardia) 569

1963. F. diphylla, Vahl

Seirpus

1964. S. lacustris, Linn.

(s. validus) 571

Fuirena

1965. I'. umbellata, Rottb. 573

Rhyncospora

1966. R. glauca, Vahl

(R. gracilis) 574

1967. R. cyperoides, Mart. 574

1968. R. aurea, Vahl

(R. surinamensis) 575

1969. R. floridn, Dietr. 575

1970. R. polyphylla, Vahl. 575

1971. R. setacea, Boeckl.

R. spermodon) 576

1972. R. mierantha, Vahl 576

Cladium

1973. C. Mariscus, $R$. Br. (C. occidentale) 573

Scleria

1974. S. pratensis, Lindl. 577

1975. S. melaleuea, Reichenb. 578

1976. S. microcarpa, Nees 578

1977. S. mitis, S'w. 578

1978. S. latifolia, $S w .578$

1979. S. flagellum, Berg. 578

1980. S. hirtella, Sw. 579

1981. S. filiformis, $S w .579$ 
Uncinia

1982. U. jamaicensis, Pers. 581

Carex

1983. C. scabrella, Wahlenb. 580 1984. C. cladostachya, Wahlenb. 580

\section{GRAMINE正.}

\section{Paspalum}

1985. P. compressum, Nees 541 1986. P. conjugatum, Berg. 541 1987. P. distichum, Linn. 541 1988. P. notatum; Fluegg. $5+1$ 1989. P. Swartzianum, Fluegg.

$$
\text { (P. filiforme) } 5+1
$$

1990. P. decumbens, $S w .542$ 1991. P, setaceum, Michx. 542 1992. P eæspitosum, Fluegg. 542 1993. P. fimbriatum, Kunth 542 1994. P. plicatulum, Michx. 542 1995. P. virgatum, Limn $5+3$ 1996. P. paniculatum, Linn. 543 1997. P. filiforme, Fluegg.

(Digitaria) 543

Eriochloa

1998. E. polystachya, H.B.K. (E. punctati) 544

Isachne

1999. I. arundinacea, Griseb. 553 2000. 1. rigens, Trin. 553

2001. I. pygmea, Griscb. 553

\section{Panicum}

2002. P. paspalodes, Pers. 54.7 2003. P. prostratum, Lam. 546 2004. P. grossarium, Linn. 546 2005. P. carthaginense, $S w .5 \pm 6$ 2006. P. fuseum, $S w, 540$ 2007. P. fasciculatum, Sw. 547 2008. P. flavercens, Sw. $5+7$ 2009. P. palmifolium, Poir. 547
2010. P. molle, $S w .547$

2011. P. zizanioides, H.B.K.

(P. oryzoides) 547

2012. P. stenodes, Griseb. 547

2013. P. laxum, Sw. 548

2014. P. distichum, Lam. 548

2015. P. pilosum, $S w .548$

2016. P. maximum, Jacq. 549

2017. P. elephantipes, Nees. 549

2018. P. hirsutum, Sw. 549

2019. P. divaricatum, Linn. 551

2020. P. Sloanei, Griseb. 551

2021. P. lanatum, $S w .551$

2022. P. sorghoideum, Hamilt. 515

2023. P. compactum, Sw. 552

2024. P. martinicense, Griseb. 5,12

2025. P. glutinosum, $S w .552$

2026. P. mierospermum, Fourn.

(P. trichanthum) 552

2027. P. brevifolium, Linn. 552

2028. P. dichotomum, Linn. 553

2029. P. acuminatum, $S w .553$

2030. P. Linkiana, Kunth

(Digitaria marginata) 544

2031. P. sanguinale, Linn.

2032. P. fimbriatum, Kunth.

(D. setigera) 544

2033. P. Myurus, Lam.

(Hymenachne) 553

3034. P. striatum, Lam.

(Hymenachne) 554

2035. P. leucophæum, H.B.K.

(Tricholæna insularis) 557

Ichnanthus

2036. I. alsinoides, Munro

(Panicum) 550

2037. I. nemorosus, Doell.

(Panicum) 550

2038. I. pallens, Doell.

(Panicum) 550

Oplismenus (Orthopogon)

2039. O. hirtellus, Roem. il

Schult. 545 


\section{List of Jamaica Flowering Plants.}

2040. O. loliaceus, Beauv. 545

2011. O. setarius, Roem. \& Schult. 545

2042. O. colonus, H.B.K. (Panicum) 545

2043. O. spectabilis, Kunth. (Panicum) 546

\section{Setaria}

2044.*S. viridis, Beauv. 554 2045. S. glauca, Beauv. 554 2046.*S. italica, Beauv. 554 2047. S. magna, Griseb. 554 2048. S. vulpiseta, Roem \& Schult. 555

2049. S. onurus, Griseb. 555 2050. S. macrostachya, H.B.K. 555 2051. S. caudata, Roem. \& Schult. 555

\section{Cenchrus}

2052. C. echinatus, Linn. 556 2053. C. tribuloides, Linn. 556

Pennisetum

2054. P. setosum, Rich. 555

Stenotaphrum

2055. S. americanum, Schrank. 544

\section{Olyra}

2056. O. latifolia, Linn. 535

2057. O. pauciflora. $S w .536$

\section{Pharus}

2058. P. latifolius, Linn. 536 2059. P. glaber, H.B.K. 536 2060. P. brasiliensis, Raddi

\section{Coix}

2061. C. Lacryma, Linn. 561.

\section{Tripsacum}

2062. T. dactyloides, Iinn.

(T. monostachyum) 557

Zea

2063. Z. Mays, Linn. 561
Leersia

2064. L. hexandra, $S w .535$

2065. L. monandra, Sw. 535

Arundinella

2066. A. martinicensis, Trin. 556

Reynaudia

2067. R. filiformis, Kunth. 534

Anthephora

2068. A. elegans, Schreb. 556

Tragus (Lappago)

2069. T. racemosus, Hall

(L. aliena) 557

Imperata

2070. I. exaltata, Brogn.

(I. caudata) 561

Erianthus

2071. E. Ravennæ, Beauv.

(E. jamaicensis) 561

Manisurus

2072. M. granularis, Linn. f. 557

Ischømum

2073. I. latifolium, Kunth

(Ischæmopogon) 560

Heteropogon

2074. H. contortus, Roem.\& Schult.

\section{Andropogon}

2075. A. saccharoides, $S w .558$

2076. A, brevifolius, $S w .558$

2077. A. gracilis, Spreng. 559

2078. A. fastigiatus, $S w .559$

2079. A. virginicus, Iinn.

(Anatherum domingense) 559

2080. A. bicornis, Linn.

(Anatherum) 559

2081. A. macrourus, Michx.

(Anatherum) 559

2082. A. squarrosus, Linn. $f$.

(Vetiveria) 559 
2083.*A. Sorghum, Brot. (Sorghum halepense) 560 2084.*A. Minarum, Kunth

\section{Aristida}

2085. A. purpurascens, Poir. 534 2086. A. setifolia, H.B.K.

Sporobolus

2087. S. virginicus, Kunth 533

2088. S. domingensis, Kunth 533 2089. S. purpuraseens, Hamilt. 532 2090. S. indicus, $R$. Br. 533

2091. S. Jacquemontii, Kunth 533

\section{Agrostis}

2092.*A. canina, Lim.

\section{Holeus}

2093.*H. lanatus, Lim.

\section{Cynodon}

2094. C. Dactylon, Per's. 540

\section{Chloris}

2095. C. crueiata, S'́. 538

2096. C. eleusinoides, Griseb. 539

2097. C. radiata, $S w .539$

2098. C. polydactyla, $S w .539$

2099. C. barbata, Sw. 539

2100. C. ciliata, $S w .539$

2101. C. petræa, Thunb. 539

\section{Bouteloua}

2102. B. litigiosa, Lag. 537

\section{Eleusine}

2103. E. indica, Gartn. 540 2104. E. ægyptiaca, Pers.

(Disctyloctenium) 540

\section{Leptochloa}

21.05. L. virgata, Becurv. 538
Gynerium

2106. G. saccharoides, Humb. \& Bonpl. 530

Arundo

2107.*A. Donax, Linn. 530

Phragmites

2108. P. martinicensis, Trin. 530

Molinia

2109.*M. corulea, Moench.

Eragrostis

2110. E. glutinosa, Trin. 532

2111. E. pilosa, Beauv. 532

2112. F. bahiensis, Schrad. 532

2113. E. poæoides, Beauv. 532

2114. E. ciliaris, Link. 532

\section{Zeugites}

2115. Z. americana, Willd. 536

Uniola

2116, U. racemiffora, Trin.

(U. virgata) 531

\section{Dactylis}

2117.*D. glomerata, Linn.

Briza

2118.*B. minor, Linn.

Poa

2119.*P. annua, Linn.

Festuca

2120.*F. bromoides, Linn.

2121. *F. elatior, Iinn.

2122.*F ovina, Linn.

\section{Bromus}

2123. B. sterilis, linn.

2124. B. unioloides, II.B.K.

\section{Chusquea}

2125. C. abietifolia, Griseb. 529

Bambusa

2126. B. vulgaris, Wendl. 5"S 


\section{List of Jamaica Flowering Plants. \\ Class III.-GYMNOSPERME $\not$.}

\begin{tabular}{|c|c|}
\hline CONIFER里. & 2129. P. Purdieanus, Hook. 504 \\
\hline Juniperus & \\
\hline 2127. J. Bermudiana, Iinn. 503 & CYCADACE $\mathbb{E}$. \\
\hline $\begin{array}{l}\text { Podocarpus } \\
\text { 2128. P. coriaceus, Rich. } 504\end{array}$ & $\begin{array}{l}\text { Zamia } \\
\text { 2130. Z. integrifolia, Ait. } 504\end{array}$ \\
\hline
\end{tabular}




\section{ADDENDA ET CORRIGENDA.}

\section{VIOLARIEA.}

Sauvagesia

51a. S. erecta, Linn.

\section{Portulaceæ.}

Talinum

73a. T. patens, Willd.

\section{Maldacee.}

\section{Sida}

104a. S. aggregata, Presl.

\section{Simarube As.}

Suriana

217a. S. maritima, Linn. 58

\section{Cyrlliere.}

Cyrilla

247a. C. antillana, Michx. 1t.,

\section{SOLANACEA.}

\section{Cestrum}

1178a. C diurnum, Linn.

Polygonacem.

Coccolobat

137t. C. uvifera, Jacq. 161
1375. C. jamaicensis, Lindau

(C. leoganensis, p.p.) 162

1376. C. leptostachyoides, Lindau

1377. C. Plumieri, Griseb. 162

1378. C. Zebra, Griseb. 162

1379. C. venosa, Griseb.

(C. diversifolia p.p. 163)

1380. C. barbadensis, Jacq.

1381. C. pirifolia, Desf.

(C. punctata, v. Jaequinii,p.p.)

1381a. C. longifolia, Fisch.

\section{Chloran'thaceE.}

Hedyosmum

1430a. H. nutans, Sw. 173

1430b. H. arborescens, She 173

LORANTHACER.

Eubrachion

1469a. E, brasiliense, Eichl.

\section{ORCHIDEF.}

Liparis

1660a. L. viridi-purpurea, Griseb.

\section{SIITAMINE/E.}

Hedychium

17Tsi.*H. coronarium, hoen.

On page 20, read Brickellia for Bricliplit.

On page 36, read Brosinum for Brosimumm.

On page 39, read Zyygupetrlum for Zyponsetrlmm.

†Substitute these names for the species of Coceolol hi on page 32 . 


\section{N D E X.}

Page.

A belmoschus

Abildgaardia

Abrus

\section{Abutilon}

Acacia -

Acalypha

A can thaceæ

A canthospermum

A cbimenes

Achras -

Achyranthes

Acidoton

Acisanthera

Acnida

Acnistus

Acrilia

Acrocomia

Acrodiclidium -

Adelia

Adelobotrys

Adenanthera

Adenostemma

㘴chmea

Atgiphila

Aeranthes

Aschynomene -

Agapanthus

Agati

Agave

A geratum

Agrostis

Albizzia

Alchornea

Aleurites

Alismace

Allamanda

Allium

Alloplectus

Aloe

Alternanthera -

Alvaradoa

Alysicarpus

A marantacex

Amarantus

Amaryllides

A maryllis

Amblogyne

Ambrosia

Ammannia

\section{4}

10

4

12

35

29

20

28

23

31

35

13

31

27
Ampelideæ

Ampelocissus

Amphistelma

Amyris

Anacardiaceæ

Anacardium

Anagallis

Anamomis

Ananas

Anatherum

Andira

Andrographis

Andropogon

Anechites

Angelonia

Anisomeles

Anoda

Anona

Anonaceæ

Anredera

Anthacanthus -

Anthephora

Anthurium

Antirrbirum

Antirrboa

Apium

A pocarpæ

A pocynacex

Aptería

Arachis

Araliaceæ

Arceuthobium

Ardisia

Arenaria

Argemone

Argitbamnia

Argyreia

Aristida

Aristolochia

Aristolochiaceæ

Aroideæ

Arpophyllum

Artanthe

Artocarpus

Arundinella

Arundo

Asclepiadeæ

Asclepias

Ascyrum
Page.

Page.

A vicennia

Ayenia

- $\mathbf{3 0}$

Baccharis 20

Bactris 43

Badiera - 3

Balanophoreæ - $\quad 34$

Bambusa $\quad$ - 48

Bassovia $\quad-27$

Bastardia

Batideæ 32

Batis - 22

Bauhinia $\quad$ - 11

Begonia $\quad 17$

Begoniaceæ 17

Beilschmiedia - $\quad 38$

Belamcanda 42

Beloperone 29

Bernardia - $\quad 35$

Besleria - 28

Bicarpellatæ $\quad 23$

Bideng - 21

Bignoniacex $\quad 29$

Bixa - - 2

Bixineæ 2

Blakea - 15

Blech'um 29

Bletia 38

Blighia - 8

Bocagea 1

Bocconia 2

Bœhmeria - 37

Boerhazvia 31

Bontia 29

Boragineæ 25

Borrera $\quad 39$

Borrichia 21

Bouchea 30

Bourreria 25

Boutelouia 48

Brachypterys 5

israssavola $\quad 39$

Brassia $\quad 39$

Brickellia 20

Briza - 48

Bromelia $\quad 41$

Bromeliaceæ 41

Bromus 48

Brosimum $\quad 36$ 
Page.

Broughtonia
Browallia
Brunella
Brunellia
Brunfelsia
Brya
Bryophyllum
Buchnera
Bucida -
Buddleia
Bulbophyllum
Bumelia
Bunchosia
Burmannia
Burmanniacea
Bursera
Burseraceae
Buxus
Byrsonima

Cacter

Cæasalpinia

Cajanns

Cakile

Calan the

Calceolaria

(valea

Calliandra

Callicarpa

Calophyllum

Calotropis

Caly cifloræ

Calycinæ

Calycogonium -

Calyptranthes -

Calyptrogyne

Calyptronoma -

Cameraria

Campanulacer

Campelia

Campylocentron

Cananga

Canavalia

Canella

Canellaceæ

Canna

Cannabis

Caperonia

(Capparidere

Capprtris

Capraria

Caprifoliaceæ

Capsella

Capsicum

Caraguata

Cardamine

Cardiospermum

Carex

Carica

Carludovica

Carum

Caryuphylleæ

Cirsearia

\section{9}

27

- 30

6
27

27
9

12

28

13

25

38

23

5

37

37

7

7

34

5

17

11

10

2

38

27

21

12

30

3

24

9

43

14

13

43

43

24

22

43

40

1

10

2

2

26

D.)

2

2

$2 x$

18

$2 \frac{2}{27}$

41

2

8

46
16

43

17

3

15
Casparea

Cassia

Cassipourea

Cassytha

Casuarina

Casuarineæ

Catalpa

Catesbæa

Catopsis

Cayaponia

Cecropia

Cedrela

Celosia

Celastrineæ

Celtis

Cenchrus

Centrosema

Centunculus

Cephaelis

Cerastium

Ceratophylleæ

Ceratophyllum

Cereus

Cestrum

Chænocephalus

Chamissoa

Chaptalia

Charianthus

Chenopodiaceæ

Chenopodium

Chevalliera

Chimarrhis

Chiococca

Chloidia

Shloranthaceæ

Chloris

Chlorophora

Chrysanthellum

Chrysanthemum

Chrysobalanus

Chrysophyllum

Chuncoa

Chnsques

Cicca

Cinnamodendron

Cinnamomum

Cionandra.

Cionosicys

Cipura

Cisf a mpelos

Cissus

Citherexylum

Citrullus

citrus

Cladium

Clematis

Cleome

Clerodendron

Clethra

Cleyera.

Clibadium

Clidemia

Clitoria
Page.

Page.

11

Clusia

Coccocypselum

Coccoloba

Cocculus

Cocos

Colia

Coffea

Coix

Cola

Colacasia.

Coleus

Colubrina

Columnea

Combretaceæ

Combretum

Commelina

Commelinaceæ -

Comocladia

Comparettia

Compositæ

Condalia

Connaracex

Conocarpus

Conostegia

Convolvulacege-

Convolvulus

Copernicia

Corchorus

Cordia

Cornaceæ

Cornutia

Coronarieæ

Corymbis

Cosmos

Costus

Cravichis

Crassulacex

Cratæra

Cremanium

Crescentia

Crinum

Critonia

Crotalaria

Croton

Cruciferæ

Crudia

Cryptarrhena

Cryptostegia

Cucumis

Cucurbitacex

Cupania

Cupbea

Curculigo

Cuscrita

Cyatbula

Cyclanthaceæ

Cynodon

Crperacere

Cyperus

Cyphomandra

Cyrilla.

- 21

14,15

Cyrilleæ

Cyrtopera

3

18

50

1

43

38

19

47

4

44

30

8
-28

13

13

43

43

8

39

20

7

9

13

14

26

26 


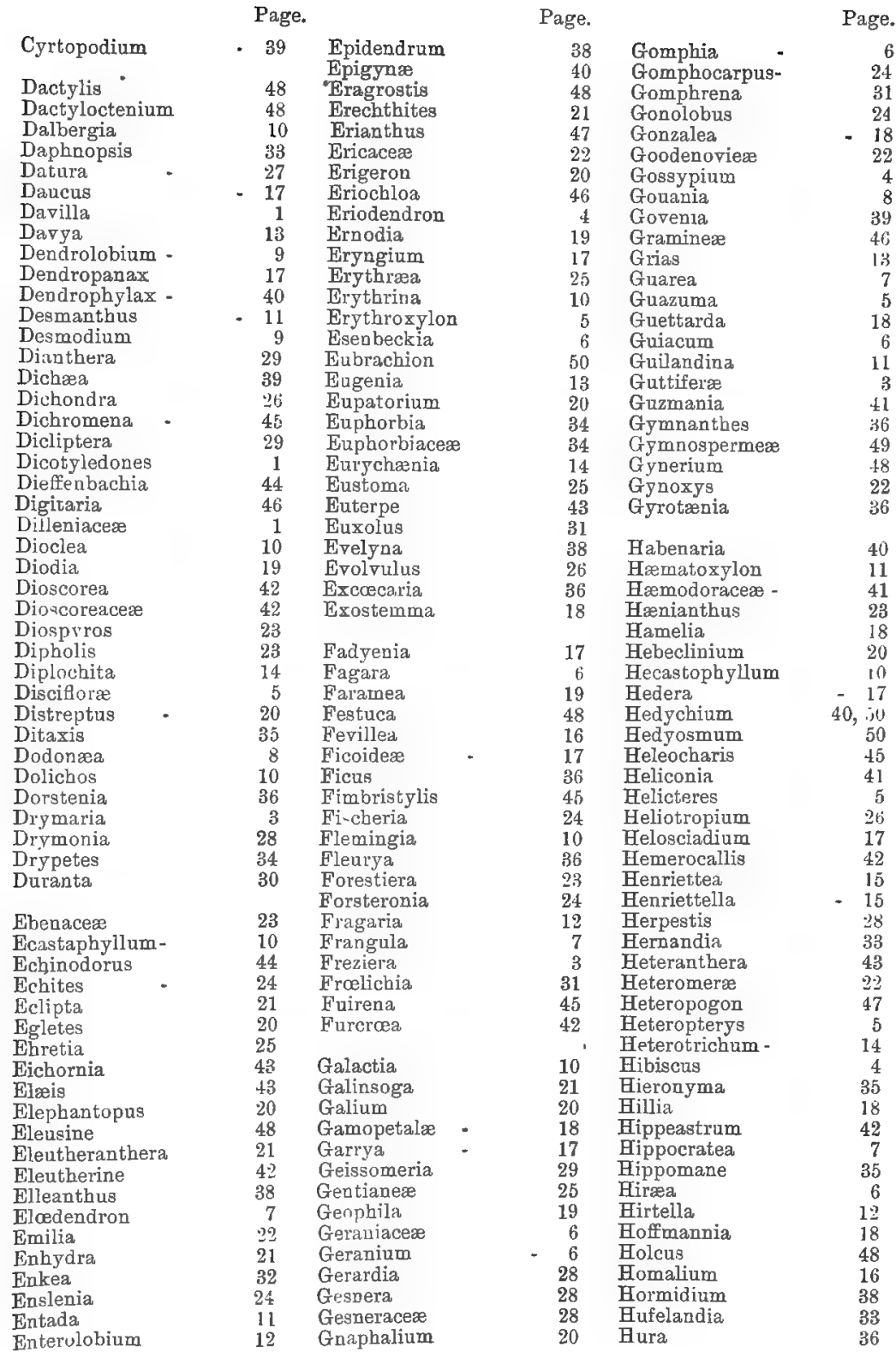




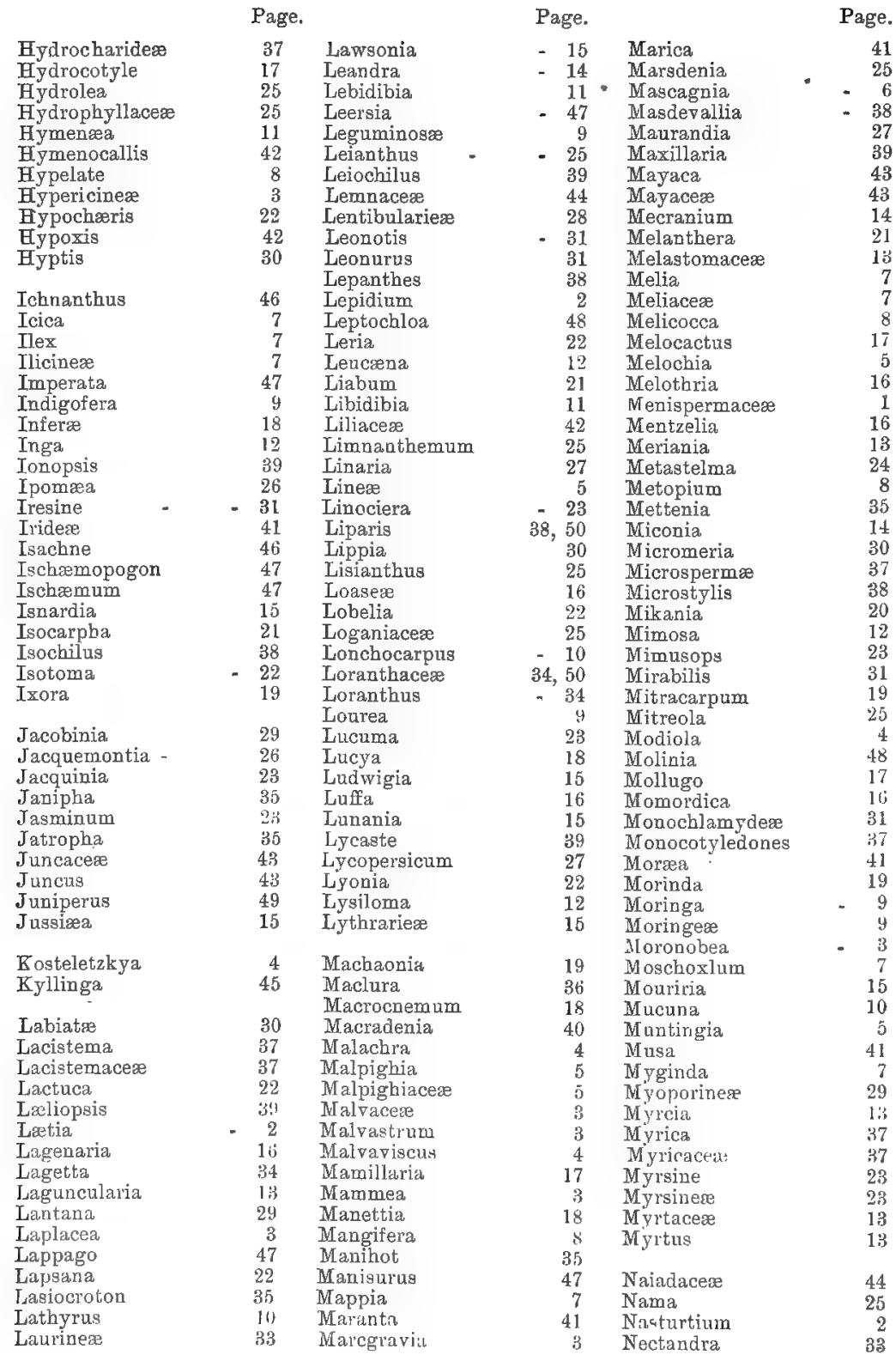


Page.

Neea

Nelumbium

Nepsera

Neptunia

Nesra

Neuroliena

Nicotiana

Nidularium

Nyctagineæ

Nymphaed

Nymphaacea

Ochroma

Ocimum .

Ocutea

Octadesmia

Octomería

Odontoglossum

Gnothera

Oriera

Olacineæ

Oldendlandia

Oleaceæ

Olyra

Omphalea

Onagrariese

Oncidium

Oplismenus

Opuntia

Orchider

Oreodoxa

Oreopanax

Ornithidium

Orthopogon

Osmanthus

Ossrea

Oxalis

Oxandra

Oxypetalum

Pachystarbys

Pachyrbizus

Palicourea

Palme

Pancratium

Panicum

Papaverncer

Pasitiom

Parkinsonia

Partheninm

Paspa um

Passiflora

Passiflorege

Pastinaca

Paullinia

Paronia

Pectis

Pedalinea

Pedilanthus

Pelexia

Peltophorum.

Peltostigma

Pennisetum
31

Pentarhaphia

Peperomia

Peleskia

Persea

Petitia

Petiveria

Petroselinum

Pencedanum

Phaius

Pharlus

Pbaseolus

Pbeuax

Philibertia

Philodendron

Pbiloxerus

\section{Phobe}

Phoradendron -

Phiagmites

Phyllanthus

Pbyllocoryne

Physalis

Physurus

Phytolacca

Phytolaccacere

Picræna

Picramnia

Picrodendron -

Pilea

Pimenta

- 17

37,50

43

Pinguicula

Piper

Piperaceæ

Piriqueta

Piscidia

Pisonia

Pistia

Pitcairnia

Pithecoctenium

Pitbecolobium

Plantagineaæ

Plantago

Platymiscium

Pleurocbænia

Plenrothallis

Plnchea

Plumbagineæ

Plumbago

Plumeria

Poa

Poducarpus

Pogronia

Polybcea

Polygala

Polygalex

Polygonacea

Polygonum

Polypetalæ

Polypremum -

Polystachya

Ponera

Pontederiacer -

Ponthieva

Porophyllum -
Page.

Page.

Portesia

Portlandia

Portulaca

Portulaceæ

Potamogeton

Prescotria

Primulaceæ

Prioria.

- 11

Priva - 30

Prosopis

Protium

Prunella

Prunus

Pseudocentrum

Pseudolmedia

Psidium

Psophocarpus

Psychotria

Pterocarpus

Pterocaulon

Pterygoloma

Punica

Pyrethrum

Quiina

Rajania

Randia

Ranuneulaceæ

Ranunculus

Ratonia

Rauwolfia

Renealmia

Reynaudia

Rhabdadenia

Rhacbicallis

Rhæo

Rhannea

Rhamnus

Rheedia.

Rbipsalis

Rhizophora

Rhizophoreæ

Rhus

Rhynocosia

Rhyncospora

Rhytidophyllum

Richardsonia

Ricinus

Rivina

Rochefortia

Rolandra

Rondeletia

3250

Rosa

Riosaceæ

Roulinia

Rourea

Rousselia

Rubiacere

Rubus

Rudgea

Ruellia
7

18

, 50

32

40

22

11

30

12

40

36

13

10

19

10

20

28

15

21

3

42

18

1

8

24

41

47

24

18

43 
Page.

\section{Rutaceæ}

Sabal

Sabicea

Sagittaria

Salicineæ

Salix

Salmea

Salvia

Samyda

Samydaces

Sapindaceæ

Sapindus

Sapium

Sapota

Sapotaceæ

Sarcomphalus

Sarcostemma

Sauroglossum

Saubagesia

Saxifrageæ

Scærola

Schæfferia

Schlegelia

Schmidelia

Schopfia

Schomburgkia -

Schradera

Schultesía

Sciadophyllum

Scirpus

Scitamineæ

Scleria

Scleropus

Scolosanthus

Scoparia

Scrophularineæ

Scybalium

Sechium

Securidaca

Senebiera

Senecio

Serjania

Sesamum

Sesbania

Sesurium

Setaria

Sida

Sideroxylon

Sikana

Sileue

Simaruba

Simarubere

Sisymbrium

Sloanea

Smilax

Solanacer

Solandra

Solanum

Sonching

Sophora

Sorgbum

Sparganophorus
6

43

18

44

37

37

21

30

16

15

8

8

35

23

23

7

24

40

50

- 12

- 22

- 7

29

8

79

39

25

17

- 45

40,50

45

31

19

28

27

34

16

3

2

22

8

29

9
17

47

4. 50

23

] 6

3

-

6,50

2

5

- 42

27,50

27

27

22

11

48

- 20
Spathelia

Specularia

Spermacoce

Spigelia

Spilanthes

Spiranthes

Spondias

Sponia

Sporobolus

Stachys

Stachytarpha

Stachytarpheta

Stelis

Stellaria

Stemodia

Stemonacanthus

Stenia

Stenoptera

Stenorrhyncus

Stenostomum

Stenotaphrum

Sterculia

Sterculiaceæ

Stigmaphyllon

Strychnodaphne

Stylosanthes

Styracex

Suriana

Swartzia

Swietenia

Syagrus

Symphonia

Symplocos

Synedrella

Syngonium

Syzygium

Tabernamontana

Talinum

Tamarindus

Tanæcium

Taraxacum

Tecoma

Tephrosia

Teramnus

Terminalia

Terustromia

Ternstrœmiaceæ

Tetracera

Tetramicra

Tetrapterys

Tetrazygia

Teucrium

Thalamifloræ

Thalassia

Theobroma

Thespesia

Theretia

Thiodia

Thrinax

Thunbergia

Thymeleaceæ

Thyrsacanthus -

Page.

Page.

6

Thyrsanthus

Tigridia

Tiliaceæ

Tillandsia

Tinantia

Tobinia

Tournefortia

Tovaria

Toromita

Tradescantia

Tragia

Tragus

Trema

Trianthema

Tribulus

Tricera

Trichilia

Tricholæna

Trifolium

Trigonidium

Trimezia

Triopterys

Tripsacum

Triumfetta

- 33

Trixis

Trophis

Turnera

Turnerace

Turpinia

Tussacia

Typba

Typhaceæ

- 24

42

41

43

6

25

3

43

35

47

36

17

6

34

7

46

9

38

42

5

47

5

22

36

16

16

8

28

43

43

Ulex

Umbelliferæ

9

17

46

Univla 48

Urechites

Urena

Urera

Urticacer

Utricularia

4

36

36

28

Vacciniaceæ 22

Vaccinium - 22

Vandeliia - $\quad 28$

Vanilla 40

Vaupellia 28

Verbena 30

Verbenaceæ 29

Verbesina 21

Verhuellia 32

Vernonia 20

Veronica $\quad$ - 28

Vetiveria 47

Viburnum $\quad 18$

Vicia 10

Vigna 10

Villamilla 32

Vinca 24

Vincetoxicum - $\quad$. 24

Viola - $\quad 2$

Violarieæ $\quad \cdot 2,50$ 
INDEX.

Page.

\begin{tabular}{|c|c|c|c|}
\hline Vitex & - & -30 & Wullschlægelia \\
\hline Vitis & & & \\
\hline Voyria & - & 25 & Xanthosoma \\
\hline & & & Ximenia \\
\hline $\begin{array}{l}\text { W allenias } \\
\text { W altheria }\end{array}$ & - & $\begin{array}{r}23 \\
5\end{array}$ & $\begin{array}{l}\text { Xiphidium } \\
\text { X plobium }\end{array}$ \\
\hline Wedelia & - & 21 & $\vec{X}$ ylopia \\
\hline Weinmannia & - & 12 & $\bar{X}$ ylosma \\
\hline Wissadula & - & 4 & \\
\hline Witheringia & & 27 & \\
\hline & & & Yucca \\
\hline
\end{tabular}

Page. Page

- 40 Zamia - 49

Zanthoxylum 6

Zea 47

44 Zebrina - 43

7 Zephyranthes 42

41 Zeugites 48

- 39 Zingiber - 40

- 1 Zinnia - 21

2 Zizyphus - 7

Zornia - 9

42 Zygopetalum $\quad \begin{gathered}39 \\ \text { Zygophylleæ }\end{gathered}$ 



Syracuse, N. $\mathbf{Y}$.

Mi. Ju 21, tow 
